\title{
Study on the Potential Active Components and Molecular Mechanism of Xiao Huoluo Pills in the Treatment of Cartilage Degeneration of Knee Osteoarthritis based on Bioinformatics Analysis and Molecular Docking Technology
}

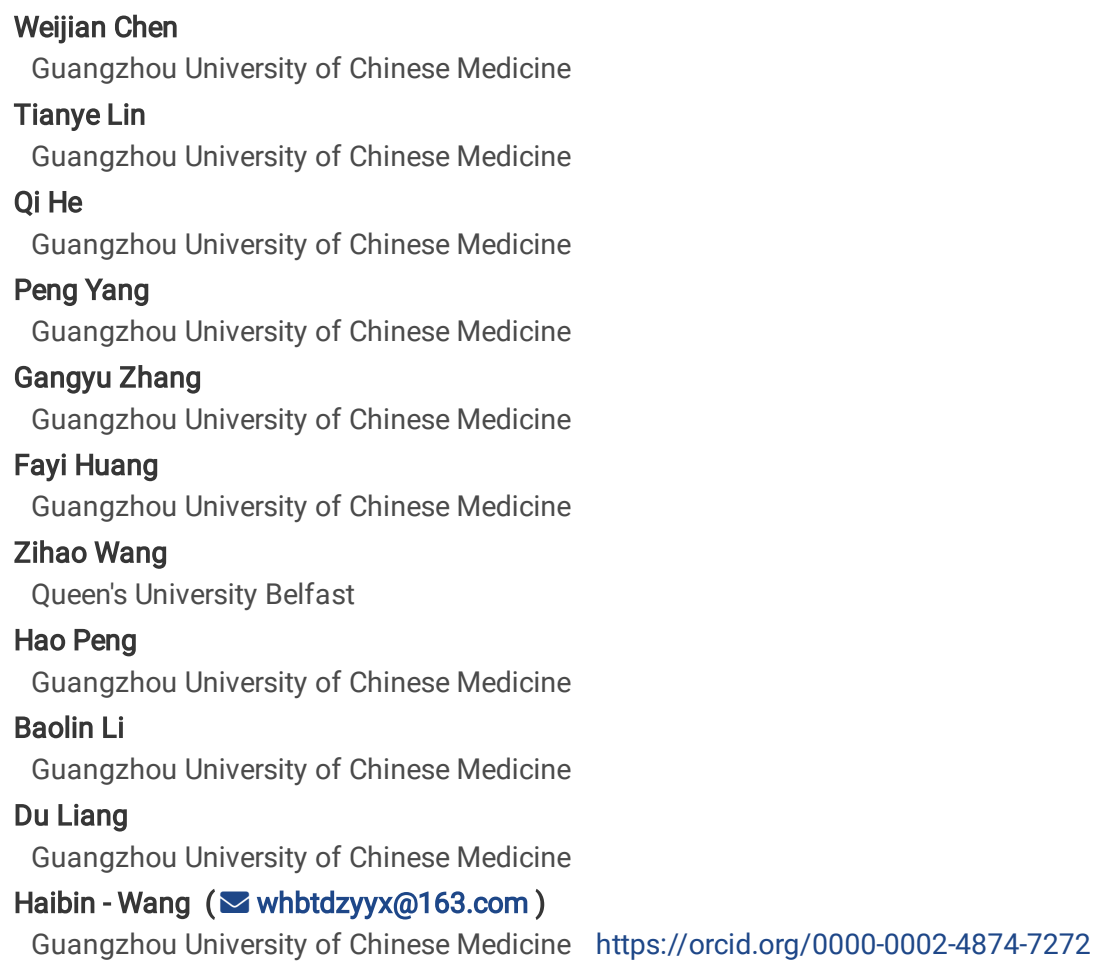

\section{Research}

Keywords: Knee osteoarthritis, XHLP, bioinformatics, network pharmacology, molecular docking

Posted Date: May 25th, 2021

DOl: https://doi.org/10.21203/rs.3.rs-404023/v2

License: (c) (1) This work is licensed under a Creative Commons Attribution 4.0 International License. Read Full License

Version of Record: A version of this preprint was published at Journal of Orthopaedic Surgery and Research on July 17 th, 2021. See the published version at https://doi.org/10.1186/s13018-021-02552-w. 


\section{Abstract}

Background: Knee osteoarthritis is a common joint degenerative disease that severely affects the quality of life. Modern pharmacological studies have confirmed that Xiao Huoluo Pills (XHLP) has anti-inflammatory, anti-oxidant, and analgesic effects. At present, it has been used to treat degenerative diseases such as osteoarthritis and hyperosteogeny. However, XHLP's specific effective ingredients and mechanism of action against osteoarthritis have not been explored. Therefore, bioinformatics technology and molecular docking technology are employed in this study to explore the molecular basis and mechanism of XHLP in the treatment of knee osteoarthritis.

Methods: Public databases (TCMSP, Batman-TCM, HERB, DrugBank, and UniProt) are used to find the effective active components and corresponding target proteins of XHLP (screening conditions: $\mathrm{OB}>30 \%, \mathrm{DL} \geq 0.18$ ). Differentially expressed genes related to cartilage lesions of knee osteoarthritis are obtained based on the GEO database (screening conditions: adjust $P$ Value $<0.01,\left|\log _{2} F C\right| \geq 1.0$ ). The Venn package in $R$ language and the BisoGenet plug-in in Cytoscape are adopted to predict the potential molecules of XHLP in the treatment of knee osteoarthritis. The XHLP-active component-target interaction network and the XHLP-knee osteoarthritis-target protein core network are constructed using Cytoscape software. Besides, GO/KEGG enrichment analysis on core genes is performed using the Bioconductor package and clusterProfiler package in the R language to explain the biological functions and signal pathways of the core proteins. Finally, molecular docking is performed through software such as Vina, LeDock, Discovery Studio 2016, PyMOL, AutoDockTools 1.5.6, so as to verify the binding ability between the active components of the drug and the core target protein.

Results: XHLP has been screened out of 71 potentially effective active compounds for the treatment of OA, mainly including quercetin, Stigmasterol, betasitosterol, Izoteolin, and ellagic acid. Knee osteoarthritis cartilage lesion sequencing data (GSE114007) was screened out of 1672 differentially expressed genes, including 913 up-regulated genes and 759 down-regulated genes, displayed as heat maps and volcano maps. Besides, 33 core target proteins are calculated by Venn data package in R and BisoGenet plug-in in Cytoscape. The enrichment analysis on these target genes revealed that the core target genes are mainly involved in biological processes such as response to oxygen levels, mechanical stimulus, vitamin, drug, and regulation of smooth muscle cell proliferation. These core target genes are involved in signaling pathways related to cartilage degeneration of knee osteoarthritis such as TNF signaling pathway and PI3K-Akt signaling pathway. Finally, the molecular docking verification demonstrates that some active components of the drug have good molecular docking and binding ability with the core target protein, further confirming that XHLP has the effect of inhibiting cartilage degeneration in knee osteoarthritis.

Conclusions: In this study, based on the research foundation of bioinformatics and molecular docking technology, the active components and core target molecules of XHLP for the treatment of cartilage degeneration of knee osteoarthritis are screened out, and the potential mechanism of XHLP inhibiting cartilage degeneration of knee osteoarthritis is deeply explored. The results provide theoretical basis and new treatment plan for XHLP in the treatment of knee osteoarthritis.

\section{Introduction}

The characteristic case changes of knee osteoarthritis (KOA) include articular cartilage degeneration, subchondral bone reactivity, osteophyte formation at the joint edge, synovial lesions, ligament laxity, and contracture[1,2]. With the aging of the population, the incidence of knee osteoarthritis is increasing year by year. Globally, osteoarthritis affects approximately 250 million people[3]. Chronic pain and disability associated with osteoarthritis (OA) can lead to anxiety, depression, and suicidal emotions. However, long-term use of non-steroidal anti-inflammatory drugs in OA patients is associated with gastrointestinal and cardiovascular side effects[4,5]. Advanced KOA is mainly treated by joint replacement surgery, which is expensive and has many postoperative complications $[6,7]$. Therefore, it is necessary to further explore the occurrence and development mechanism of KOA, and provide new theoretical basis and diagnosis and treatment guidelines for the precise treatment of KOA.

Traditional Chinese medicine has been used to treat KOA for many years. Starting from the local application of knee joint medicine and the whole body, it can effectively relieve pain symptoms, improve joint function, and protect joint structure[8, 9]. XHLP comes from the classic traditional Chinese medicine "Taiping Huimin He Jifang Prescription", composed of six herbs: Chuanwu, Caowu, Dilong, Araceae, Frankincense, and Myrrh[10]. At present, it is mainly used to treat diseases such as rheumatoid arthritis, hyperosteosis, osteoarthritis, and stroke hemiplegia[11]. Modern pharmacological studies have confirmed that XHLP has the effects of anti-inflammatory, anti-oxidant damage, analgesia, blood vessel expansion, inhibiting platelet aggregation, and improving immunity[12]. Besides, the diester-type diterpene alkaloids of the active extracts of Radix Aconiti and Radix Aconitum play anti-inflammatory effects by inhibiting the process of prostaglandin metabolism[13] and chemotaxis of leukocytes mediated by chemokines[14]. Seo et al.[15] revealed that the antibacterial peptide lumbricusin of earthworm extract can significantly reduce the inflammatory reaction in vivo and in vitro caused by LPS such as COX-2, IL-6, and TNF-a[16]. By inhibiting the activation of neutrophil product NF-KB, frankincense extract can down-regulate the expression of inflammatory factors TNF-a, IL-1, and INF-y, alleviate inflammation[17, 18]. Meanwhile, it can inhibit the activity of collagenase MMP-1 and alleviate cartilage degeneration[19]. Therefore, XHLP is expected to have a strong anti-osteoarthritis effect. However, the potential pharmacological mechanism of XHLP and its interaction with osteoarthritis-related targets and pathways still need to be further investigated.

The traditional Chinese herbal medicine treatment KOA has passed the test of long-term medical practice. However, the "multi-component" and "multifunctional" of Chinese medicine has not been recognized by the world because its specific mechanism of action remains unclear[20]. Network pharmacology can reveal the complex network relationship between drugs, targets, and diseases through high-throughput screening, network visualization, and network topology analysis, and accurately predict and analyze the mechanism of action of traditional Chinese medicine compounds[21]. It has the characteristics of "multi-component, multi-target, multi-pathway", turning the complex into simple. Additionally, potential differentially expressed genes are analyzed and discovered by using the genome sequencing data of KOA patients[22, 23], providing strong evidence for

Page 2/22 
the biological targets and potential mechanisms of XHLP in the treatment of KOA. This study aims to use bioinformatics analysis and molecular docking technology to clarify and verify the effective active components, targets, and potential mechanisms of XHLP in knee osteoarthritis.( Figure 1)

\section{Methods}

\section{XHLP's effective active components screening and identification}

XHLP is composed of six traditional Chinese medicines: Chuanwu, Caowu, Dilong, Araceae, Frankincense, and Myrrh, with the composition ratio of 1.2:1.2:1.2:1.2:1.0:1.0. XHLP's constituent medicines "Chuanwu", "Caowu", "Dilong", "Araceae", "Frankincense", and "Myrrh" were entered into the TCM pharmacology system database[24] (TCMSP: traditional Chinese medicine system pharmacology,https://tcmspw.com/tcmsp.php) in turn for search. Oral Bioavailability $(\mathrm{OB})$ refers to the speed and degree of absorption of oral drugs into the circulation of the body (including the bioavailability and utilization rate of the drug). Drug-Likeness (DL) is a concept based on the physical and chemical properties and molecular structure of existing drugs, used to evaluate the similarity between a compound and a known drug. According to the common screening criteria of the TCMSP database and previous literature results $[25,26]$, the active components with $\mathrm{OB}>30 \%$ and $\mathrm{DL} \geq 0.18$ were selected for subsequent analysis and research.

\section{Target protein screening and drug-active component-target network construction}

The TCMSP database is used to search for the target of the active components of the drugs obtained by screening, and all the target protein names obtained from the screening of the TCMSP database are imported into the DrugBank database (https://www.drugbank.ca/) and the UniProt database (https://www.uniprot.org). The species is set as Homo sapiens. Comparison and gene name correction are performed on the obtained target protein, converting into the target gene name and deleting the target protein without corresponding gene name. The obtained active component of the medicine and the active component establish a corresponding relationship with the target. The drug-active component-target interaction network model is obtained by Cytoscape 3.7.2 software[27]. The "Networkanalyze" function in Cytoscape 3.7.2 software[28] is employed to perform network topology analysis, screening out core active components and potential target genes.

\section{Obtaining gene expression profile of human osteoarthritis cartilage tissue}

The high-throughput sequencing data of cartilage tissue genes related to osteoarthritis are downloaded in the GEO (gene expression omnibus) database (https://www.ncbi.nlm.nih.gov/geo/). The screening conditions are: 1) osteoarthritis; 2) human; 3) cartilage tissue. The whole-genome sequencing data GSE114007 that meets the screening criteria are obtained, including 18 normal knee cartilage tissue samples from K-L I patients and 20 knee cartilage tissue samples from K-L IV KOA patients. All the above sequencing data sets are downloaded based on the GEO database of GPL11154 and GPL18573 platforms. The general clinical information of patients is presented in Table 1.

Table 1. Characteristics of study subjects.

\begin{tabular}{|c|c|c|c|c|c|}
\hline \multirow[t]{2}{*}{ GSE114007 } & \multirow[t]{2}{*}{ Number of subjects } & \multirow[t]{2}{*}{ Age (years, mean $\pm S D$ ) } & \multicolumn{2}{|l|}{ Sex } & \multirow[t]{2}{*}{ K-L Stage } \\
\hline & & & Male & Female & \\
\hline Normal & 18 & $36.61 \pm 13.08$ & 13 & 5 & $\otimes(n=18)$ \\
\hline Osteoarthritis & 20 & $66.20 \pm 7.16$ & 8 & 12 & $\nabla(n=20)$ \\
\hline
\end{tabular}

\section{Acquisition and analysis of differential genes in cartilage tissue of osteoarthritis}

The original sequencing data of GSE114007 are downloaded from the GEO database. The FastQC software is adopted to perform quality assessment and quality control processing on the original data. Besides, the Hisat2 software is used to select a reference genome to compare and analyze the clean data obtained after quality control processing. Then, the number of gene reads is quantitatively analyzed using the HTSeq Python package to obtain the count file. Next, the counts data obtained by sorting are filtered. Filter conditions are: 1 ) variance filtering; 2 ) low abundance filtering. Besides, the data are standardized using the $\log _{2}$-counts per million method. The DESeq2 package in $\mathrm{R}$ language is employed to perform difference analysis and calculate the difference genes between the two groups. Set $P<0.01$. adjust $P$ Value $<0.01$ indicates that the change range is greater than or equal to twice $\left|\log _{2} F C\right| \geq 1.0$ as the criterion for screening differential genes. Among them, $\log _{2} F C \geq 1.0$ and $\log _{2} F C \leq-1.0$ represent up-regulation and down-regulation of gene expression, respectively. Finally, the differentially expressed genes in cartilage tissue of the osteoarthritis group and the healthy control group were derived, namely KOA differentially expressed genes (DEGs). The online analysis website ClustVis[29] (https://biit.cs.ut.ee/clustvis/) is adopted to draw the heat map and cluster analysis of the differential genes obtained by screening. Then, the adjust $\mathrm{P}$ Value in the processed data is converted to -log ${ }_{10}$. According to $\log _{2} \mathrm{FC}$, $-\log _{10}$ (adjust $\mathrm{P}$ Value) is divided into up-regulated genome, down-regulated genome, no statistical difference, and non-expressed difference genome. The processed data are imported into GraphPad Prism 7 to draw a volcano map.

\section{Intersection and interactive network construction of active component of medicine and osteoarthritis target}

The Venn diagram of the common target genes in the target of the active component of the drug and the target of the disease is displayed through the online Venn diagram production website InteractiVenn (http://www.interactivenn.net/)[30]. The obtained common target genes are imported into the online analysis website STRING[31] to construct protein-protein interaction (PPI). Besides, the BisoGenet plug-in in the Cytoscape 3.7.2 software[32] is 
used to construct the target PPI of the drug active component and the PPI network of the osteoarthritis differential gene. The "Networkanalyze" function in Cytoscape 3.7.2 software is used for network topology analysis. The PPI and KOA differential gene PPI networks of the active pharmaceutical ingredient are combined to obtain the PPI network shared by the active pharmaceutical ingredient PPI and the KOA differential gene PPI network. The CytoNCA plugin in the Cytoscape 3.7.2 software is adopted to calculate the multi-center network topology for the PPI shared by the target of the active component of the drug and the KOA differential gene. Then, screening is conducted according to the median value of Degree, BetweennessCentrality (BC), ClosenessCentrality (CC), LAC, and NeighborhoodConnectivity (NC) values. It is concluded that the active components of XHLP act on the potential core target genes of KOA.

\section{Enrichment analysis of target gene by GO/KEGG}

To further explore the potential mechanism of XHLP active components on KOA and the function of common target genes, we use the Bioconductor package and clusterProfiler package[33] in the R language to perform gene ontology (GO) and KEGG (Kyoto Encyclopedia of Genes and Genomes) signal pathway analysis on the target genes obtained in the previous step. The GO analysis mainly includes cellular Component (CC), molecular Function (MF), and biological Process (BP) of potential target genes. Meanwhile, the Pathview package is used to draw the corresponding signal path diagram.

\section{Molecular docking verification of the binding of the active component of the drug to the target protein}

The molecular docking verification of XHLP and potential targets is conducted to explore the interaction between them. The structural formula of the active components of XHLP is downloaded from the TCMSP database (https://tcmspw.com/tcmsp.php). The Chem3D software is used to make 3D structures of effective active components. Then, the 3D structure of the core protein gene is downloaded from the PDB database (http://www.rcsb.org/). The PyMOL software is employed to perform operations such as dewatering and phosphate removal of proteins. Besides, the AutoDockTools 1.5 .6 software[34] is adopted to convert the pdb format of active components and core protein gene files into pdbqt format and search for active pockets. Finally, the Vina script[35] and LeDock software[36] are applied to calculate the molecular binding energy and display the results of molecular docking. Simultaneously, Discovery Studio 2016 is used to find the docking site and calculate the LibDockScore[37, 38]. Next, the output molecular docking results are imported into PyMOL software for molecular docking verification display. Binding energy < 0 indicates that the ligand and the receptor can bind spontaneously. The binding energy $\leq-5.0 \mathrm{kcal} \cdot \mathrm{mol}^{-1}$ is selected as the screening basis to evaluate the reliability of biological information analysis and prediction[39].

\section{Results}

\section{Screening of effective active components of XHLP}

A total of 568 active components of XHLP were retrieved through TCMSP database and Batman-TCM database, including 12 species of Radix Aconiti, 27 species of Radix Aquilinum, 123 species of Araceae, 127 species of Frankincense, and 276 species of Myrrh. However, Earth Dragon failed to retrieve the active components in the TCMSP database and Batman-TCM database. Therefore, three active components of Earthworm were obtained through literature search and HERB (A high-throughput experiment- and reference-guided database of traditional Chinese medicine,http://herb.ac.cn/) identification by the herbal group. Under the screening conditions of $O B \geq 30 \%$ and $D L \geq 0.18,71$ effective active compounds were selected from the active components of XHLP, including 3 species of Radix Aconiti and 8 species of Radix Aconiti, 3 species of Earth Dragon, 7 species of Araceae, 8 species of Frankincense, and 45 species of Myrrh; besides, there is 1 common active component of Radix Aconiti and Radix Aquilinum, and 2 common active components of Araceae and Myrrh (Table 2).

\section{Table 2 XHLP effective active component information}




\begin{tabular}{|c|c|c|c|c|c|}
\hline ID & $\begin{array}{l}\text { Pubchem } \\
\text { Cld }\end{array}$ & Component & $\begin{array}{l}\text { OB } \\
(\%)\end{array}$ & $\mathrm{DL}$ & $\begin{array}{l}\text { Herbs } \\
\text { (Latin name) }\end{array}$ \\
\hline CHW1 & 22212681 & $\begin{array}{l}\text { 1-[(5R,8R,9S,10S,12R,13S,14S,17S)-12-hydroxy-10,13-dimethyl- } \\
2,3,4,5,6,7,8,9,11,12,14,15,16,17 \text {-tetradecahydro-1 H-cyclopenta[a]phenanthren-17- } \\
\text { yl]ethanone }\end{array}$ & 33.47 & 0.42 & Aconiti Radix \\
\hline CHW2 & 92979 & delta4,16-Androstadien-3-one & 37.63 & 0.31 & \\
\hline CAW1 & 133323 & Izoteolin & 39.53 & 0.51 & \multirow{7}{*}{$\begin{array}{l}\text { Aconitum } \\
\text { Kusnezoffii } \\
\text { Reichb }\end{array}$} \\
\hline CAW2 & 441742 & karakoline & 51.73 & 0.73 & \\
\hline CAW3 & 21598997 & 3-deoxyaconitine & 30.96 & 0.24 & \\
\hline CAW4 & 21599000 & 3-acetylaconitine & 37.05 & 0.2 & \\
\hline CAW5 & 157539 & crassicauline A & 34.13 & 0.21 & \\
\hline CAW6 & 155569 & yunaconitine & 33.56 & 0.2 & \\
\hline CAW7 & 441749 & napelline & 34.48 & 0.72 & \\
\hline DIL1 & - & lumbrifebrine & - & - & \multirow[t]{3}{*}{ Pheretima } \\
\hline DIL2 & - & lumbritin & - & - & \\
\hline DIL3 & - & lumbrolysin & - & - & \\
\hline TLX1 & 22524484 & $\begin{array}{l}{[(2 \mathrm{R})-2-[[[(2 \mathrm{R})-2-(\text { benzoylamino)-3-phenylpropanoyl]amino]methyl]-3-phenylpropyl] }} \\
\text { acetate }\end{array}$ & 38.88 & 0.56 & \multirow{5}{*}{$\begin{array}{l}\text { Arisaematis } \\
\text { Rhizoma }\end{array}$} \\
\hline TLX2 & 5283637 & 24-epicampesterol & 37.58 & 0.71 & \\
\hline TLX3 & 12303645 & sitosterol & 36.91 & 0.75 & \\
\hline TLX4 & 5997 & CLR & 37.87 & 0.68 & \\
\hline TLX5 & 5364473 & 8,11,14-Docosatrienoic acid, methyl ester & 43.23 & 0.3 & \\
\hline RUX1 & 101257 & tirucallol & 42.12 & 0.75 & \multirow[t]{8}{*}{ Olibanun } \\
\hline RUX2 & 15181201 & 0-acetyl-a-boswellic acid & 42.73 & 0.7 & \\
\hline RUX3 & 637234 & 3alpha-Hydroxy-olean-12-en-24-oic-acid & 39.32 & 0.75 & \\
\hline RUX4 & 168928 & Boswellic acid & 39.55 & 0.75 & \\
\hline RUX5 & 44559813 & phyllocladene & 33.4 & 0.27 & \\
\hline RUX6 & - & 3-oxo-tirucallic,acid & 42.86 & 0.81 & \\
\hline RUX7 & 15181201 & acetyl-alpha-boswellic,acid & 42.73 & 0.7 & \\
\hline RUX8 & 44583885 & incensole & 45.59 & 0.22 & \\
\hline MOY1 & 13258914 & quercetin-3-O- $\beta$-D-glucuronide & 30.66 & 0.74 & \multirow[t]{14}{*}{ Myrrha } \\
\hline MOY2 & 5281855 & ellagic acid & 43.06 & 0.43 & \\
\hline MOY3 & 440832 & pelargonidin & 37.99 & 0.21 & \\
\hline MOY4 & 5283663 & poriferasta-7,22E-dien-3beta-ol & 42.98 & 0.76 & \\
\hline MOY5 & 69232409 & guggulsterol-VI & 54.72 & 0.43 & \\
\hline MOY6 & 128211 & mansumbinoic acid & 48.1 & 0.32 & \\
\hline MOY7 & 57401582 & myrrhanol C & 39.96 & 0.58 & \\
\hline MOY8 & - & (8R)-3-oxo-8-hydroxy-polypoda -13E,17E,21-triene & 44.83 & 0.59 & \\
\hline MOY9 & 102242792 & myrrhanones B & 34.39 & 0.67 & \\
\hline MOY10 & - & epimansumbinol & 61.81 & 0.4 & \\
\hline MOY11 & 51407984 & diayangambin & 63.84 & 0.81 & \\
\hline MOY12 & 667495 & (2R)-5,7-dihydroxy-2-(4-hydroxyphenyl)chroman-4-one & 42.36 & 0.21 & \\
\hline MOY13 & - & (13E,17E,21E)-8-hydroxypolypodo-13,17,21-trien-3-one & 44.34 & 0.58 & \\
\hline MOY14 & - & (13E,17E,21E)-polypodo-13,17,21-triene-3,18-diol & 39.96 & 0.58 & \\
\hline
\end{tabular}

Page 5/22 


\begin{tabular}{|c|c|c|c|c|c|}
\hline MOY15 & - & 16-hydroperoxymansumbin-13(17)-en-3ß-ol & 41.05 & 0.49 & \\
\hline MoY16 & - & mansumbin-13(17)-en- 3,16-dione & 41.78 & 0.45 & \\
\hline MoY17 & - & (16S, 20R)-dihydroxydammar-24-en-3-one & 37.34 & 0.78 & \\
\hline MoY18 & 5318259 & 15a-hydroxymansumbinone & 37.51 & 0.44 & \\
\hline MOY19 & 636531 & 28-acetoxy-15a-hydroxymansumbinone & 41.85 & 0.67 & \\
\hline MOY20 & 101281214 & isofouquierone & 40.95 & 0.78 & \\
\hline MOY21 & 11004967 & $\begin{array}{l}{[(5 \mathrm{aS}, 8 \mathrm{aR}, 9 \mathrm{R})-8-0 \times 0-9-(3,4,5-\text { trimethoxyphenyl)-5,5a,6,9-tetrahydroisobenzofurano[6,5-f] }} \\
{[1,3] \text { benzodioxol-8a-yl] acetate }}\end{array}$ & 44.08 & 0.9 & \\
\hline MOY22 & - & phellamurin_qt & 56.6 & 0.39 & \\
\hline MOY23 & - & $(3 R, 20 S)-3,20$-dihydroxydammar- 24-ene & 37.49 & 0.75 & \\
\hline MOY24 & - & 3-methoxyfuranoguaia-9- en-8-one & 35.15 & 0.18 & \\
\hline MOY25 & 6439929 & Guggulsterone & 42.45 & 0.44 & \\
\hline MOY26 & 441774 & petunidin & 30.05 & 0.31 & \\
\hline MOY27 & 5280343 & quercetin & 46.43 & 0.28 & \\
\hline MOY28 & - & 4,17(20)-(cis)-pregnadiene-3,16-dione & 51.42 & 0.48 & \\
\hline MOY29 & 101297585 & Guggulsterol IV & 33.59 & 0.74 & \\
\hline MoY30 & 10496532 & $\begin{array}{l}\text { (7S,8R,9S,10R,13S,14S,17Z)-17-ethylidene-7-hydroxy-10,13-dimethyl- } \\
\text { 1,2,6,7,8,9,11,12,14,15-decahydrocyclopenta[a]phenanthrene-3,16-dione }\end{array}$ & 35.75 & 0.48 & \\
\hline MOY31 & - & $7 \beta, 15 \beta$ - dihydroxypregn-4-ene-3,16-dione & 43.11 & 0.51 & \\
\hline MOY32 & - & 11a-hydroxypregna-4,17(20)-trans-diene-3,16-dione & 36.62 & 0.47 & \\
\hline MoY33 & 102242791 & myrrhanone A & 40.25 & 0.63 & \\
\hline MOY34 & - & $3 \beta$-acetoxy-16ß,20(R)-dihydroxydammar-24-ene & 38.72 & 0.81 & \\
\hline MOY35 & - & 1a-acetoxy-9,19-cyclolanost-24-en-3ß-ol & 44.4 & 0.78 & \\
\hline MOY36 & 12019028 & $\begin{array}{l}\text { [(3R,5R,8R,9R,10R,13R,14R,17S)-17-[(2S,5S)-5-(2-hydroxypropan-2-yl)-2-methyloxolan- } \\
\text { 2-yl]-4,4,8,10,14-pentamethyl-2,3,5,6,7,9,11,12,13,15,16,17-dodecahydro-1H- } \\
\text { cyclopenta[a]phenanthren-3-yl] acetate }\end{array}$ & 33.07 & 0.8 & \\
\hline MOY37 & 21625900 & cabraleone & 36.21 & 0.82 & \\
\hline MOY38 & - & 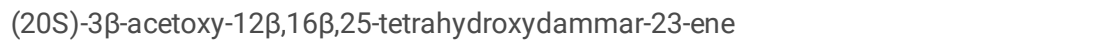 & 34.89 & 0.82 & \\
\hline MOY39 & - & 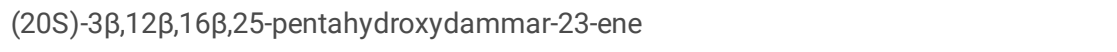 & 37.94 & 0.75 & \\
\hline MOY40 & - & 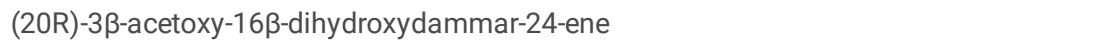 & 40.36 & 0.82 & \\
\hline MOY41 & - & $3 \beta$ - hydroxydammar-24-ene & 40.27 & 0.82 & \\
\hline MOY42 & 10870920 & $\begin{array}{l}\text { [(5S,6R,8R,9Z)-8-methoxy-3,6,10-trimethyl-4-oxo-6,7,8,11-tetrahydro-5H- } \\
\text { cyclodeca[b]furan-5-yl] acetate }\end{array}$ & 34.76 & 0.25 & \\
\hline MOY43 & - & 2-methoxyfuranoguaia-9-ene-8-one & 66.18 & 0.18 & \\
\hline A1 & 441737 & hypaconitine & 31.39 & 0.26 & $\begin{array}{l}\text { Aconiti } \\
\text { Radix/Aconitum } \\
\text { Kusnezoffii } \\
\text { Reichb }\end{array}$ \\
\hline B1 & 222284 & beta-sitosterol & 36.91 & 0.75 & $\begin{array}{l}\text { Arisaematis } \\
\text { Rhizoma/Myrrha }\end{array}$ \\
\hline B2 & 5280794 & Stigmasterol & 43.83 & 0.76 & $\begin{array}{l}\text { Arisaematis } \\
\text { Rhizoma/Myrrha }\end{array}$ \\
\hline
\end{tabular}

\section{Construction and analysis of XHLP-active component-target network}

The 71 active components in XHLP were entered into the TCMSP database and Batman-TCM database to find their corresponding target proteins. The 206 target proteins obtained were imported into the DrugBank database and the UniProt database for comparison and correction, and 170 standard gene names were output. Next, the XHLP composition and effective active components and the corresponding relationship between the active components and the target was obtained. The XHLP-active component-target network was constructed using Cytoscape 3.7.2 software. The network has 248 nodes, 
including 6 drug nodes, 71 active compound nodes, and 170 target nodes, with a total of 506 edges (Figure 2). Each edge in the network represents the active component contained in the drug and the interaction between the active component and the target gene. The "Networkanalyze" function in the Cytoscape 3.7.2 software is employed to perform topology analysis and calculation on the network. Among them, Degree value and BetweennessCentrality value (BC) are the key parameters to measure the nodes in the network. The active components in this network have an average relationship with 6 targets, and each target has an interconnection relationship with an average of 2.5 active components. Therefore, the active components in XHLP may be related to multiple targets while one target may be related to multiple active components. It can be revealed by analyzing the Degree value and BC value of the active component node and target gene node in the network that the top 5 active molecules are MOY27-quercetin, B2Stigmasterol, B1-beta-sitosterol, CAW1-Izoteolin, and MOY2-ellagic acid, which can be connected to 136, 53, 53, 18, and 17 target proteins, respectively; the top six targets are PGR, NCOA2, NR3C2, PTGS2, PTGS1, and RXRA, which can interact with 25, 24, 18, 18, 12 , and 12 active components, respectively (Table 3).

Table 3 Degree and BC values of the top 8 effective active components and targets of XHLP

\begin{tabular}{|c|c|c|c|c|c|}
\hline Type & Description & Degree & $\mathrm{BC}$ & Link & Rank \\
\hline \multirow[t]{8}{*}{ Component } & quercetin & 136 & 0.71368345 & 136 & 1 \\
\hline & Stigmasterol & 52 & 0.08655831 & 52 & 2 \\
\hline & beta-sitosterol & 52 & 0.07340369 & 52 & 3 \\
\hline & Izoteolin & 18 & 0.07627614 & 18 & 4 \\
\hline & ellagic acid & 17 & 0.03680757 & 17 & 5 \\
\hline & $\begin{array}{l}{[(5 \mathrm{aS}, 8 \mathrm{aR}, 9 \mathrm{R})-8-0 \times 0-9-(3,4,5 \text {-trimethoxyphenyl)-5,5a,6,9-tetrahydroisobenzofurano[6,5-f] }} \\
{[1,3] \text { benzodioxol-8a-yl] acetate }}\end{array}$ & 16 & 0.03229396 & 16 & 6 \\
\hline & pelargonidin & 12 & 0.01540433 & 12 & 7 \\
\hline & 3-methoxyfuranoguaia-9- en-8-one & 9 & 0.00568861 & 9 & 8 \\
\hline \multirow[t]{8}{*}{ Gene } & PGR & 25 & 0.02750561 & 25 & 1 \\
\hline & NCOA2 & 24 & 0.07571674 & 24 & 2 \\
\hline & PTGS2 & 18 & 0.04966347 & 18 & 3 \\
\hline & NR3C2 & 18 & 0.00563037 & 18 & 4 \\
\hline & RXRA & 12 & 0.03377059 & 12 & 5 \\
\hline & PTGS1 & 12 & 0.03359142 & 12 & 6 \\
\hline & CHRM3 & 10 & 0.00861658 & 10 & 7 \\
\hline & CHRM1 & 10 & 0.00440313 & 10 & 8 \\
\hline
\end{tabular}

\section{Acquisition and analysis of differentially expressed genes in KOA cartilage tissue}

Our research is based on 20 cases of cartilage tissue samples from KOA patients ( 8 male cases, 12 female cases, aged 66.20 \pm 7.16 , and K-L IV patients accounted for 20 cases) and 18 cases of normal cartilage tissue samples (aged $36.61 \pm 13.08,13$ male cases and 5 female cases) from the sequencing data set GSE114007. The general clinical characteristics of the patients are presented in Table 1. The filter conditions are: Variance filter=15.0 and Low abundance=4.0; the standardization method is $\log _{2}$-counts per millio. Box diagram and PCA diagram of the data filtering and before and after standardization are illustrated in Figure 3.A.B. P Value $<0.01$ and expression changes greater than or equal to twice $\left(\mid \log _{2} \mathrm{FCl} \geq 1.0\right)$ were the criteria for screening differential genes. In the GSE114007 data set, 1672 differentially expressed genes were screened, including 913 up-regulated genes and 759 down-regulated genes. A heat map was drawn for the DEGs selected in GSE114007 and the top 50 DEGs with the most significant differences in the adjust $P$ Value selection (Figure 3.C.D). Among them, red and green represent up-regulation and down-regulation of gene expression. Then, -log 10 conversion was performed on the adjust $P$ Value of the gene after the differential analysis process. According to $\log _{2}$ FC, - $\log _{10}$ (adjust $P$ Value) is divided into up-regulated genome, down-regulated genome, and no statistically different genome). The results were imported into GraphPad Prism 7.0 to draw a volcano map (Figure 3.E).

\section{XHLP effective active component-KOA intersection target analysis and mutual PPI network construction}

The 170 drug active component targets obtained above and the 1672 differentially expressed genes obtained from the differential analysis of the sequencing data set GSE114007 were introduced into the online Venn diagram production website InteractiVenn. Then, a total of 33 potential targets for XHLP treatment of KOA cartilage changes were obtained by matching mapping. The Venn diagram of the effective active component-KOA intersection

Page $7 / 22$ 
target is exhibited in Figure 4 A. Besides, 33 potential target genes were imported into STRING online analysis website (https://string-db.org/); hide unconnected targets were set, and "medium confidence $>0.400$ " in the lowest interaction score was determined; the result data of protein-protein interaction were exported (Figure 4.B.C). Next, the PPI network of potential target genes was obtained by Cytoscape 3.7.2 software, and the cytoHubba plug-in was adopted to screen Hub genes[40]. Two different algorithms, Degree and Betweenness, were employed to the top 6 potential core target genes: VEGFA, CCND1, MYC, JUN, MMP9, and MMP2 (Figure 4.D.E.F). Meanwhile, the BisoGenet plug-in in the Cytoscape 3.7.2 software was applied to construct the PPI network of the drug active component target and the KOA differential gene PPI network (Figure 5.A.B). The two PPI networks were merged and mapped to an intersection network (Figure 5.C). The network topology of the intersection network was analyzed and calculated using CytoNCA to obtain parameter values such as Degree, Betweenness, BetweennessCentrality (BC), ClosenessCentrality (CC), NeighborhoodConnectivity (NC), and LAC. The first screening threshold was Degree>66 (that is, twice the median value of Degree). The result revealed that there are 1,170 nodes and 52,739 edges in the secondary network graph (Figure 5.D). The second screening thresholds were Degree $>109$, Betweenness $>460.887, B C>0.00043, C C>0.4421, N C>146.685$, and $L A C>16.999$. The results indicated that there are 144 nodes and 1723 edges in the three-level network graph, which also included genes such as VEGFA, CCND1, MYC, JUN, MMP9, and MMP2 (Figure 5.E).

\section{Enrichment analysis of intersection target by GO/KEGG}

The Bioconductor package and clusterProfiler package in R language were used to perform GO and KEGG pathway enrichment analysis on 33 XHLP active components-KOA intersection targets. The $\mathrm{GO}$ analysis of 33 potential target genes demonstrated that the biological process (BP) mainly focuses on response to oxygen levels, mechanical stimulus, vitamin, drug, and regulation of smooth muscle cell proliferation; cellular component (CC) is mainly concentrated in collagen-containing extracellular matrix, endoplasmic reticulum lumen, and fibrillar collagen trimer; molecular function (MF) is mainly manifested in activating transcription factor binding, growth factor binding, and core promoter sequence-specific DNA binding (Table 4, Figure 6 A.B.C). The enrichment analysis indicated that the KEGG pathway mainly focuses on AGE-RAGE signaling pathway in diabetic complications, Relaxin signaling pathway, TNF signaling pathway, PI3K-Akt signaling pathway, Fluid shear stress and atherosclerosis signaling pathways. The pathview package was adopted to display the signal pathway diagram related to cartilage (Figure 6.D.E.F.G).

Table 4 Annotation of intersection target gene GO/KEGG enrichment analysis 


\begin{tabular}{|c|c|c|c|c|c|}
\hline Category & Term & Description & Count & P.adj.value & Genes \\
\hline \multirow[t]{5}{*}{$\mathrm{BP}$} & GO:0070482 & $\begin{array}{l}\text { response to } \\
\text { oxygen levels }\end{array}$ & 11 & 0.00000005 & PTGS2,PLAU,VEGFA,CDKN1A,MMP2,PPARG,MYC,ICAM1,COL1A1,SLC2A4,HK2 \\
\hline & G0:0009612 & $\begin{array}{l}\text { response to } \\
\text { mechanical } \\
\text { stimulus }\end{array}$ & 8 & 0.00000150 & PTGS2,JUN,RELA,PPARG,FOS,COL1A1,COL3A1,IRF1 \\
\hline & G0:0033273 & $\begin{array}{l}\text { response to } \\
\text { vitamin }\end{array}$ & 6 & 0.00000436 & PTGS2,RELA,PPARG,CCND1,COL1A1,SPP1 \\
\hline & GO:0048660 & $\begin{array}{l}\text { regulation of } \\
\text { smooth } \\
\text { muscle cell } \\
\text { proliferation }\end{array}$ & 7 & 0.00000436 & PTGS2,JUN,CDKN1A,MMP2,MMP9,PPARG,IGFBP3 \\
\hline & Go:0042493 & $\begin{array}{l}\text { response to } \\
\text { drug }\end{array}$ & 9 & 0.00000436 & PTGS2,RELA,CDKN1A,PPARG,CCND1,FOS,MYC,ICAM1,COL1A1 \\
\hline \multirow[t]{5}{*}{$\mathrm{CC}$} & G0:0062023 & $\begin{array}{l}\text { collagen- } \\
\text { containing } \\
\text { extracellular } \\
\text { matrix }\end{array}$ & 6 & 0.00580234 & MMP2,MMP9,ICAM1,COL1A1,COL3A1,PCOLCE \\
\hline & GO:0005788 & $\begin{array}{l}\text { endoplasmic } \\
\text { reticulum } \\
\text { lumen }\end{array}$ & 5 & 0.00580234 & PTGS2,COL1A1,COL3A1,SPP1,IGFBP3 \\
\hline & G0:0005583 & $\begin{array}{l}\text { fibrillar } \\
\text { collagen trimer }\end{array}$ & 2 & 0.00580234 & COL1A1,COL3A1 \\
\hline & G0:0098643 & $\begin{array}{l}\text { banded } \\
\text { collagen fibril }\end{array}$ & 2 & 0.00580234 & COL1A1,COL3A1 \\
\hline & GO:0005667 & $\begin{array}{l}\text { transcription } \\
\text { regulator } \\
\text { complex }\end{array}$ & 5 & 0.01219532 & JUN,RELA,PPARG,CCND1,FOS \\
\hline \multirow[t]{5}{*}{ MF } & Go:0033613 & $\begin{array}{l}\text { activating } \\
\text { transcription } \\
\text { factor binding }\end{array}$ & 5 & 0.00005068 & JUN,RELA,PPARG,FOS,MYC \\
\hline & GO:0019838 & $\begin{array}{l}\text { growth factor } \\
\text { binding }\end{array}$ & 5 & 0.00035415 & COL1A1,COL3A1,INSR,IGFBP3,ERBB3 \\
\hline & GO:0001046 & $\begin{array}{l}\text { core promoter } \\
\text { sequence- } \\
\text { specific DNA } \\
\text { binding }\end{array}$ & 3 & 0.00456064 & RELA,FOS,MYC \\
\hline & GO:0005178 & $\begin{array}{l}\text { integrin } \\
\text { binding }\end{array}$ & 4 & 0.00456064 & IGF2,ICAM1,COL3A1,SPP1 \\
\hline & Go:0004955 & $\begin{array}{l}\text { prostaglandin } \\
\text { receptor } \\
\text { activity }\end{array}$ & 2 & 0.00456064 & PPARG,PTGER3 \\
\hline \multirow{7}{*}{ KEGG } & hsa05219 & Bladder cancer & 7 & 0.00000002 & VEGFA,CDKN1A,MMP2,MMP9,CCND1,MYC,RASSF1 \\
\hline & hsa05205 & $\begin{array}{l}\text { Proteoglycans } \\
\text { in cancer }\end{array}$ & 10 & 0.00000015 & PLAU,VEGFA,CDKN1A,MMP2,MMP9,IGF2,CCND1,MYC,COL1A1,ERBB3 \\
\hline & hsa04933 & $\begin{array}{l}\text { AGE-RAGE } \\
\text { signaling } \\
\text { pathway in } \\
\text { diabetic } \\
\text { complications }\end{array}$ & 8 & 0.00000015 & JUN,RELA,VEGFA,MMP2,CCND1,ICAM1,COL1A1,COL3A1 \\
\hline & hsa04926 & $\begin{array}{l}\text { Relaxin } \\
\text { signaling } \\
\text { pathway }\end{array}$ & 8 & 0.00000087 & JUN,RELA,VEGFA,MMP2,MMP9,FOS,COL1A1,COL3A1 \\
\hline & hsa05167 & $\begin{array}{l}\text { Kaposi } \\
\text { sarcoma- } \\
\text { associated } \\
\text { herpesvirus } \\
\text { infection }\end{array}$ & 9 & 0.00000095 & PTGS2,JUN,RELA,VEGFA,CDKN1A,CCND1,FOS,MYC,ICAM1 \\
\hline & hsa04668 & $\begin{array}{l}\text { TNF signaling } \\
\text { pathway }\end{array}$ & 7 & 0.00000479 & PTGS2,JUN,RELA,MMP9,FOS,ICAM1,IRF1 \\
\hline & hsa04151 & $\begin{array}{l}\text { PI3K-Akt } \\
\text { signaling } \\
\text { pathway }\end{array}$ & 10 & 0.00001120 & RELA,VEGFA,CDKN1A,IGF2,CCND1,MYC,COL1A1,INSR,SPP1,ERBB3 \\
\hline
\end{tabular}

Page 9/22 


\section{Molecular docking verification}

The top 5 active molecules in the 2.2 results (quercetin, Stigmasterol, beta-sitosterol, Izoteolin, and ellagic acid) and the top 6 potential core target genes in the 2.4 results (VEGFA, CCND1, MYC, JUN, MMP9, and MMP2) were selected for molecular docking verification. The Chem3D software was employed to draw the corresponding 3D structure according to the structural formulas of the 5 effective active components, and output in mol*2 format. The 3D structures of 6 core proteins were downloaded from the PDB database and output in pdb format. The AutoDockTools 1.5.6 software was used to convert active components and core proteins into pdbqt format to find active pockets, that is, the ligand is combined with one or more amino acid residues to form $\mathrm{H}$ bonds, $\mathrm{H}-\pi$ bonds, $\mathrm{H}-\pi$ bonds, or $\pi-\pi$ bonds and other active sites. Vina script, LeDock, and Discovery Studio 2016 software were operated to calculate the binding energy of ligand and receptor. The results suggested that quercetin can form a stable docking model with CCND1, JUN, MMP9, and MMP2, respectively; beta-sitosterol can dock with JUN and MMP2 protein ligands; izoteolin and ellagic acid can form stable docking with JUN, MMP9, and MMP2 protein ligands (Table 5). When the receptor-ligand binding energy calculated by Vina and LeDock was $\leq-5.0 \mathrm{kcal}^{\circ} \mathrm{mol}{ }^{-1}$ and DS can find the docking site, the results of the active component receptor output by the LeDock software were imported into Pymol, and the 3D molecular docking display was performed with the protein ligand (Figure 7). Simultaneously, the Discovery Studio 2016 software was adopted to display the results of molecular docking that meet the requirements in two dimensions (Figure 8).

Table 5 Comparison of binding energy of molecular docking Vina, LeDock, and DS

\begin{tabular}{|c|c|c|c|c|c|}
\hline \multirow[b]{2}{*}{ Compound } & \multirow[b]{2}{*}{ Strecture } & \multirow[b]{2}{*}{ Threct(higand) } & \multicolumn{3}{|c|}{ Birding Znary } \\
\hline & & & $\begin{array}{c}\text { Ving } \\
\text { (kcalomort) }\end{array}$ & $\begin{array}{l}\text { InDock } \\
\text { (kent-mor') }\end{array}$ & $\begin{array}{c}\text { DS } \\
\text { (hitidoctiscoro) }\end{array}$ \\
\hline \multirow{6}{*}{ quercetix } & & VEGFA(STSY) & -6.00 & -5.09 & - \\
\hline & & CCNDI(2W96) & -6.30 & -5.59 & 90.3571 \\
\hline & & MYC(SI42) & -8.00 & -7.70 & \\
\hline & & JUN(3V3v) & -8.80 & -6.60 & 99.4514 \\
\hline & & MMP9(GESM) & -9.90 & -6.86 & 145.044 \\
\hline & & $\operatorname{MMP2(4WK} 7)$ & -9.50 & -6.82 & 138.262 \\
\hline \multirow{6}{*}{ Stigmaster ol } & & VEGFA(5TS9) & -4.90 & -4.08 & \\
\hline & & CCNDI (2W96) & -650 & 4.00 & 87.8871 \\
\hline & & MYC(SL42) & -6.40 & -5.38 & . \\
\hline & & $\operatorname{suN(3V3V)}$ & -720 & 4.86 & 107.282 \\
\hline & & MMPS(6ESM) & -8.50 & -5.12 & - \\
\hline & & MMP2(4WK7) & -5.80 & -4.98 & 117.641 \\
\hline \multirow{6}{*}{ bets-sitosterol } & & VEGFA(STYY) & -5.70 & -4.25 & - \\
\hline & & CCNDI(2W96) & -6.40 & -4.27 & - \\
\hline & & MYC(SLA2) & -6.30 & -5.53 & - \\
\hline & & JUN(3V3V) & -950 & -5.23 & 109.886 \\
\hline & & MMP9(6ESM) & -8.80 & -5.57 & - \\
\hline & & $\operatorname{MMPZ(4WK} 7)$ & -7.40 & -5.14 & 124.419 \\
\hline \multirow{6}{*}{ Iroteolin } & & VEGFA(STS89) & -5.50 & -4.02 & - \\
\hline & & CCNDI(2W96) & -5.70 & 4.70 & - \\
\hline & & $\mathrm{MYC}(5442)$ & -6.10 & -5.95 & \\
\hline & & JUN(3V3V) & -8.80 & -5.89 & 96.172 \\
\hline & & MMPG(6ESM) & -7.70 & -5.27 & 117.762 \\
\hline & & $\operatorname{MMP2(4WK} 7)$ & -830 & -5.86 & 118.482 \\
\hline \multirow{6}{*}{ ellagic acid } & & VEGFA(STSY) & -6.10 & 4.38 & \\
\hline & & CCNDI(2W96) & -6.30 & -4.92 & - \\
\hline & & MYC(SLA2) & -630 & -6.44 & - \\
\hline & & $\operatorname{suN(3V3v)}$ & -9.50 & -6.40 & 100.529 \\
\hline & & MMP9(6ESM) & -7.70 & -5.47 & 132.727 \\
\hline & & $\operatorname{MMP} 2(4 \mathrm{WK} 7)$ & -8.60 & -5.80 & 120.031 \\
\hline
\end{tabular}

\section{Discussion}

Bioinformatics was first proposed by Dr. Li Huaan in 1978. People gradually realized the importance of combining computer science with biology. With the continuous surge of experimental data and biological information (about doubling every 15 months), information management and analysis has become an urgent problem to be solved. Therefore, data mining can help us find valuable information from massive amounts of data and convert it into meaningful knowledge. At present, life science research is transforming from experimental analysis combined with data accumulation to data analysis to guide experimental verification. Network pharmacology and bioinformatics analysis can be based on massive experimental data and clinical trial results, contributing to exploring the potential mechanism of TCM treatment and prevention of diseases, especially for the research on the complex chemical components of Chinese herbal medicine and the multi-target and multi-mechanism of diseases[41]. Studies have discovered that some of the extracts in the XHLP composition of drugs play an essential role in the treatment of KOA. However, the specific treatment mechanism has not been thoroughly studied. Therefore, based on bioinformatics analysis and mining, this study explored the potential effective active components, molecular targets, and 
mechanism of action of XHLP in the treatment of KOA cartilage degeneration, and further used molecular docking technology to verify the binding ability of the effective active components in XHLP with molecular targets. It fully verified the molecular basis of XHLP treatment of KOA and provided a theoretical basis for clinical treatment of KOA. Besides, bioinformatics analysis demonstrated that the potential mechanism of XHLP in the treatment of KOA cartilage degeneration has the features of "multi-component, multi-target, multi-pathway".

In this study, the XHLP-active component-target network was constructed. The network topology analysis revealed that the potential active components of XHLP treatment KOA mainly include quercetin, Stigmasterol, beta-sitosterol, Izoteolin, and ellagic acid. Hu et al.[42] discovered that quercetin can inhibit the inflammation and apoptosis of chondrocytes and provide cartilage cells with an environment promoting cartilage formation to enhance cartilage repair in the OA environment. Gabay[43] observed that Stigmasterol is a plant sterol that binds to chondrocyte membranes, can reduce the expression of MMP related to cartilage degradation and the pro-inflammatory mediator PGE2 by inhibiting the NF-KB pathway, and has the properties of antiinflammatory and inhibiting cartilage matrix catabolism. KIM et al.[44] proposed that in high-fat diet-induced intestinal inflammation, beta-sitosterol can inactivate NF-KB by interfering with the binding between LPS and TLR4 to reduce the production of pro-inflammatory cytokines. Besides, Lin et al.[45] demonstrated that ellagic acid can inhibit inflammation caused by IL-1 $\beta$ stimulation, reduce the expression of MMP-13, ADAMTS5, COX-2 and INOS in human OA chondrocytes, and weaken IL-1 $\beta$-induced inflammation by inhibiting the NF-KB pathway. There is no related research on Izoteolin in KOA. Therefore, the effective active components in XHLP may inhibit cartilage cell apoptosis, reduce cartilage matrix catabolism, and impede the NF-KB pathway to lower the production of pro-inflammatory cytokines, playing a synergistic effect in slowing down the progression of KOA cartilage degeneration.

Through the mutual mapping of the XHLP target and the differentially expressed genes of KOA, 33 potential molecular targets were obtained. Furthermore, Cytohubba, BisoGenet, and CytoNCA plug-in in Cytoscape software were used to obtain 6 potential therapeutic targets for XHLP treatment of KOA, namely VEGFA, CCND1, MYC, JUN, MMP9, and MMP2. A large number of studies have revealed that the expression of VEGFA is elevated in patients with advanced OA[46-48]. VEGFA can up-regulate the expression of MMP-1, MMP-3, and MMP-13 in chondrocytes and down-regulate the expression of key extracellular matrix components (including aggrecan and type II collagen) to accelerate the process of KOA cartilage degeneration. Zan et al.[49] discovered that CCND1 gene silencing can enhance the inhibitory effect on IL-1ß-induced proliferation of OA chondrocytes. Zou et al.[50] put forward that silencing the C-myc gene can reduce the effect of IL-1 $\beta$ on the cycle progression and apoptosis of chondrocytes in OA rats. Rhee et al.[51] found that IL-1 $\beta$ activates the expression of JUN in articular chondrocytes, inhibiting the expression of SOX9 and type II collagen in chondrocytes and the anabolic reaction of the extracellular matrix, accelerating the damage of arthritic chondrocytes. Studies have suggested that MMP-9 exacerbates local inflammation by increasing the expression of pro-inflammatory mediators such as TNF-a and IL-1, leading to accelerated degeneration of cartilage and overgrowth of subchondral bone[52,53]. Besides, MMP-2 can effectively lyse and inactivate monocyte chemotactic protein 3 (MCP-3). This not only prevents the inflammatory response from starting but also removes inflammatory factors such as TNF-a and IL-17 in the body, preventing the further development of inflammation[54]. Therefore, the above targets may all play a role in the development of KOA cartilage degeneration and are potential targets for XHLP to treat KOA.

The enrichment analysis of target genes GO/KEGG indicated that "Oxygen levels", "mechanical stimulus", "collagen-containing extracellular matrix", "TNF signaling pathway", "PI3K-Akt signaling pathway", and "Fluid shear stress" may be a potential way of XHLP treatment of KOA. Many studies[55-57] have revealed that the mechanical stress environment of joints is a significant factor affecting the activity of cartilage cells in the body, and accelerates the catabolism of osteoarthritis cartilage by interacting with pro-inflammatory factors. TNF-a can inhibit the synthesis of matrix macromolecules, stimulate the synthesis and secretion of cartilage degrading enzymes, prostaglandin (PGE2), and promote the synthesis of inflammatory factors, leading to cartilage destruction, increased inflammation, and finally degeneration of KOA cartilage[58, 59]. Moreover, the activation of PI3K/AKT signaling pathway can inhibit chondrocyte apoptosis and promote the proliferation of chondrocytes to slow down the process of KOA cartilage degeneration[60, 61]. Meanwhile, the effective active components in XHLP have the characteristics of inhibiting chondrocyte apoptosis, reducing cartilage matrix catabolism, and eliminating the production of pro-inflammatory cytokines. Therefore, XHLP can play a role in inhibiting chondrocyte apoptosis, anti-inflammatory, and other mechanisms to treat or delay the degeneration of KOA cartilage.

Finally, the binding ability of the active components of XHLP with molecular target domains was verified in this study through molecular docking technology. The results demonstrated that quercetin can form a stable docking model with CCND1, JUN, MMP9, and MMP2, respectively; beta-sitosterol can dock with JUN and MMP2 protein ligands; Izoteolin and ellagic acid can form stable docking with JUN, MMP9, and MMP2 protein ligands. According to the calculations with Vina, LeDock, and DS software, quercetin can form the most stable molecular docking with the 6ESM ligand of MMP9 protein; MMP9 is a pro-inflammatory protein, and can exert a direct anti-inflammatory effect to treat KOA cartilage degeneration when combined with quercetin, which is the effective active component in XHLP. However, this study still has some limitations.First, the results of this study lack cell and animal experimental verification.Another limitation is the false negative due to the targets rooted from a different database, which may have biased impact because of different experimental conditions. We are also aware of the public databases that have limited information.

\section{Conclusion}

In this study, the potentially effective active components, molecular targets, and action pathways of XHLP in the treatment of KOA cartilage degeneration were analyzed using bioinformatics. Besides, 71 effective active components were discovered for potential treatment of KOA; 1672 differentially expressed genes in cartilage degeneration with KOA were discovered through bio-information analysis. Mutual mapping was used to obtain 33 potential targets for the XHLP treatment of KOA. This study discovered 33 molecular targets such as VEGFA, CCND1, MYC, JUN, MMP9, and MMP2. These molecular targets are associated with the functions of "inflammatory response", "mechanical stimulus", "TNF signaling pathway", and "PI3K-Akt signaling 
pathway", involved in signaling pathways related to inflammation and chondrocyte apoptosis. Moreover, molecular docking verification was conducted to verify that the active components of XHLP can form a stable docking model with potential molecular targets. To sum up, the XHLP treatment of KOA plays a synergistic role in anti-inflammatory and inhibiting chondrocyte apoptosis through the characteristics of "multi-component,multi-target, multipathway".

\section{Abbreviations}

OA:osteoarthritis,KOA:Knee osteoarthritis,XHLP:Xiao Huoluo Pills,TCMSP:traditional Chinese medicine system pharmacology,OB:Oral Bioavailability,DL:Drug-Likeness,GEO:gene expression omnibus,DEGs:differentially expressed genes,PPI:protein-protein interaction, BC: Betweenness Centrality,NC:Neighborhood Connectivity,GO:gene ontology,KEGG:Kyoto Encyclopedia of Genes and Genomes,CC:cellular Component,MF:molecular Function,BP:biological Process

\section{Declarations}

\section{Acknowledgement}

Grateful acknowledgement is made to my supervisor Professor Liang Du and Wang haibin who gave me considerable help by means of suggestion, comments and criticism. In addition, I deeply appreciate the contribution to this thesis made in various ways by my friends and classmates.

\section{Author contributions}

C.W.J and L.T.Y was responsible for the design and implementation of the study presented. C.W.J and H.Q was responsible for the inclusions and literature review. Y.P, Z.G.Y, H.F.Y,W.Z.H,P.H and L.B.L was responsible for the acquisition of the data. C.W.J and L.T.Y prepared the initial draft of the manuscript. L.D and W.H.B gave critical feedback during the study and critically revised the submitted manuscript for important intellectual content. All authors have read and approved the final manuscript to be submitted.

\section{Competing interests}

The authors declare that they have no competing interests.

\section{Funding}

This research was financially supported by National Natural Science Foundation of China (No.81774339;No.82074462) to Wang Haibin.

\section{Ethical approval}

This study was conducted in agreement with the Declaration of Helsinki and its later amendments or comparable ethical standards and had been approved by the ethics board of The First Affiliated Hospital of Guangzhou University of Chinese Medicine (No: Y®2020ख119).

\section{Availability of data and materials}

All the data will be available upon motivated request to the corresponding author of the present paper.

\section{Consent for publication}

Not applicable.

\section{References}

[1] Cross M, Smith E, Hoy D, Nolte S, Ackerman I, Fransen M, Bridgett L, Williams S, Guillemin F, Hill CL et al: The global burden of hip and knee osteoarthritis: estimates from the global burden of disease 2010 study. ANN RHEUM DIS 2014, 73(7):1323-1330.

[2] Kim JR, Yoo JJ, Kim HA: Therapeutics in Osteoarthritis Based on an Understanding of Its Molecular Pathogenesis. INT J MOL SCI 2018, 19(3).

[3] Tang X, Wang S, Zhan S, Niu J, Tao K, Zhang Y, Lin J: The Prevalence of Symptomatic Knee Osteoarthritis in China: Results From the China Health and Retirement Longitudinal Study. ARTHRITIS RHEUMATOL 2016, 68(3):648-653.

[4] Latourte A, Kloppenburg M, Richette P: Emerging pharmaceutical therapies for osteoarthritis. NAT REV RHEUMATOL 2020, 16(12):673-688.

[5] UK NCGC: Osteoarthritis: Care and Management in Adults. London: National Institute for Health and Care Excellence (UK); 2014.

[6] Glyn-Jones S, Palmer AJ, Agricola R, Price AJ, Vincent TL, Weinans H, Carr AJ: Osteoarthritis. LANCET 2015, 386(9991):376-387.

[7] Hochberg MC, Altman RD, April KT, Benkhalti M, Guyatt G, McGowan J, Towheed T, Welch V, Wells G, Tugwell P: American College of Rheumatology 2012 recommendations for the use of nonpharmacologic and pharmacologic therapies in osteoarthritis of the hand, hip, and knee. Arthritis Care Res (Hoboken) 2012, 64(4):465-474. 
[8] Yang M, Jiang L, Wang Q, Chen H, Xu G: Traditional Chinese medicine for knee osteoarthritis: An overview of systematic review. PLOS ONE 2017, 12(12):e189884.

[9] Wang L, Zhang XF, Zhang X, Guo DY, Duan YW, Wang ZC, Pei LS, Ru H, Cheng JX, Shi YJ et al: Evaluation of the Therapeutic Effect of Traditional Chinese Medicine on Osteoarthritis: A Systematic Review and Meta-Analysis. PAIN RES MANAG 2020, 2020:5712187.

[10] The State Pharmacopoeia Committee of People's Republic of China. Pharmacopoeia of People's Republic of China. Vol. 1. In., vol. pp. 40-41, 58,127,193,233,247-248.: Chemical Industry Press; Beijing, China: 2020.

[11] Zhou Y, Cui Y, Liu Y, Shen G: [Bioavailability study on xiaohuoluo pills]. Zhongguo Zhong Yao Za Zhi 1995, 20(3):159-161, 191.

[12] Pan J XLZD: Immunosuppresive, antioxidative, anti-inflammatory and analgesic effect of Xiahuoluo pills. Chinese Journal of Clinical Rehabilitation 2006(2006(47):183-188.).

[13] Cui P, Han H, Wang R, Yang L: Identification and determination of Aconitum alkaloids in Aconitum herbs and Xiaohuoluo pill using UPLC-ESI-MS. MOLECULES 2012, 17(9):10242-10257.

[14] Kiss T, Orvos P, Bánsághi S, Forgo P, Jedlinszki N, Tálosi L, Hohmann J, Csupor D: Identification of diterpene alkaloids from Aconitum napellus subsp. firmum and GIRK channel activities of some Aconitum alkaloids. FITOTERAPIA 2013, 90:85-93.

[15] Seo M, Lee JH, Baek M, Kim MA, Ahn MY, Kim SH, Yun EY, Hwang JS: A novel role for earthworm peptide Lumbricusin as a regulator of neuroinflammation. Biochem Biophys Res Commun 2017, 490(3):1004-1010.

[16] Li C, Chen M, Li X, Yang M, Wang Y, Yang X: Purification and function of two analgesic and anti-inflammatory peptides from coelomic fluid of the earthworm, Eisenia foetida. PEPTIDES 2017, 89:71-81.

[17] Ge CY, Zhang JL: Bioactive sesquiterpenoids and steroids from the resinous exudates of Commiphora myrrha. NAT PROD RES 2019, 33(3):309-315.

[18] Liang Y LPHQ: Acetyl-11-keto-beta-boswellic acid regulates the activities of matrix metalloproteinases-1,-2,-9. Chinese Journal of Pathophysiolog 2009(2009, 25(10):2004-2011.).

[19] Ammon HP: Modulation of the immune system by Boswellia serrata extracts and boswellic acids. PHYTOMEDICINE 2010, $17(11): 862-867$.

[20] Li S, Zhang B: Traditional Chinese medicine network pharmacology: theory, methodology and application. Chin J Nat Med 2013, 11(2):110-120.

[21] Lee WY, Lee CY, Kim YS, Kim CE: The Methodological Trends of Traditional Herbal Medicine Employing Network Pharmacology. Biomolecules 2019, $9(8)$.

[22] Santolini M, Barabási AL: Predicting perturbation patterns from the topology of biological networks. Proc Natl Acad Sci U S A 2018, 115(27):E6375E6383.

[23] Chen S, Kang J, Xing Y, Zhao Y, Milton DK: Estimating large covariance matrix with network topology for high-dimensional biomedical data.

Computational Statistics and Data Analysis 2018, 127.

[24] Ru J, Li P, Wang J, Zhou W, Li B, Huang C, Li P, Guo Z, Tao W, Yang Y et al: TCMSP: a database of systems pharmacology for drug discovery from herbal medicines. J Cheminform 2014, 6:13.

[25] Wan Y, Xu L, Liu Z, Yang M, Jiang X, Zhang Q, Huang J: Utilising network pharmacology to explore the underlying mechanism of Wumei Pill in treating pancreatic neoplasms. BMC Complement Altern Med 2019, 19(1):158.

[26] Xu X, Zhang W, Huang C, Li Y, Yu H, Wang Y, Duan J, Ling Y: A novel chemometric method for the prediction of human oral bioavailability. INT J MOL SCI 2012, 13(6):6964-6982.

[27] Shannon P, Markiel A, Ozier O, Baliga NS, Wang JT, Ramage D, Amin N, Schwikowski B, Ideker T: Cytoscape: a software environment for integrated models of biomolecular interaction networks. GENOME RES 2003, 13(11):2498-2504.

[28] Assenov Y, Ramírez F, Schelhorn SE, Lengauer T, Albrecht M: Computing topological parameters of biological networks. BIOINFORMATICS 2008, 24(2):282-284.

[29] Metsalu T, Vilo J: ClustVis: a web tool for visualizing clustering of multivariate data using Principal Component Analysis and heatmap. NUCLEIC ACIDS RES 2015, 43(W1):W566-W570.

[30] Heberle H, Meirelles GV, Da SF, Telles GP, Minghim R: InteractiVenn: a web-based tool for the analysis of sets through Venn diagrams. BMC BIOINFORMATICS 2015, 16(1):169. 
[31] Szklarczyk D, Gable AL, Lyon D, Junge A, Wyder S, Huerta-Cepas J, Simonovic M, Doncheva NT, Morris JH, Bork P et al: STRING v11: protein-protein association networks with increased coverage, supporting functional discovery in genome-wide experimental datasets. NUCLEIC ACIDS RES 2019, 47(D1):D607-D613.

[32] Martin A, Ochagavia ME, Rabasa LC, Miranda J, Fernandez-de-Cossio J, Bringas R: BisoGenet: a new tool for gene network building, visualization and analysis. BMC BIOINFORMATICS 2010, 11:91.

[33] Yu G, Wang LG, Han Y, He QY: clusterProfiler: an R package for comparing biological themes among gene clusters. OMICS 2012, $16(5): 284-287$.

[34] Trott O, Olson AJ: AutoDock Vina: improving the speed and accuracy of docking with a new scoring function, efficient optimization, and multithreading. J COMPUT CHEM 2010, 31(2):455-461.

[35] Nguyen NT, Nguyen TH, Pham T, Huy NT, Bay MV, Pham MQ, Nam PC, Vu VV, Ngo ST: Autodock Vina Adopts More Accurate Binding Poses but Autodock4 Forms Better Binding Affinity. J CHEM INF MODEL 2020, 60(1):204-211.

[36] Cao H, Sun Y, Wang L, Zhao C, Fu J, Zhang A: Understanding the microscopic binding mechanism of hydroxylated and sulfated polybrominated diphenyl ethers with transthyretin by molecular docking, molecular dynamics simulations and binding free energy calculations. MOL BIOSYST 2017, 13(4):736-749.

[37] Zhang L, Shi X, Huang Z, Mao J, Mei W, Ding L, Zhang L, Xing R, Wang P: Network Pharmacology Approach to Uncover the Mechanism Governing the Effect of Radix Achyranthis Bidentatae on Osteoarthritis. BMC Complement Med Ther 2020, 20(1):121.

[38] Zhang J, Zhang Q, Chen X, Liu Y, Xue J, Dahan A, Zhang H, Chai Y: Revealing Synergistic Mechanism of Multiple Components in Gandi Capsule for Diabetic Nephropathy Therapeutics by Network Pharmacology. Evid Based Complement Alternat Med 2018, 2018:6503126.

[39] Veber DF, Johnson SR, Cheng HY, Smith BR, Ward KW, Kopple KD: Molecular properties that influence the oral bioavailability of drug candidates. J MED CHEM 2002, 45(12):2615-2623.

[40] Chin $\mathrm{CH}$, Chen SH, Wu HH, Ho CW, Ko MT, Lin CY: cytoHubba: identifying hub objects and sub-networks from complex interactome. BMC SYST BIOL 2014, 8 Suppl 4(Suppl 4):S11.

[41] Huang C, Zheng C, Li Y, Wang Y, Lu A, Yang L: Systems pharmacology in drug discovery and therapeutic insight for herbal medicines. BRIEF BIOINFORM 2014, 15(5):710-733.

[42] Hu Y, Gui Z, Zhou Y, Xia L, Lin K, Xu Y: Quercetin alleviates rat osteoarthritis by inhibiting inflammation and apoptosis of chondrocytes, modulating synovial macrophages polarization to M2 macrophages. Free Radic Biol Med 2019, 145:146-160.

[43] Gabay O, Sanchez C, Salvat C, Chevy F, Breton M, Nourissat G, Wolf C, Jacques C, Berenbaum F: Stigmasterol: a phytosterol with potential antiosteoarthritic properties. Osteoarthritis Cartilage 2010, 18(1):106-116.

[44] Kim KA, Lee IA, Gu W, Hyam SR, Kim DH: $\beta$-Sitosterol attenuates high-fat diet-induced intestinal inflammation in mice by inhibiting the binding of lipopolysaccharide to toll-like receptor 4 in the NF-KB pathway. MOL NUTR FOOD RES 2014, 58(5):963-972.

[45] Lin Z, Lin C, Fu C, Lu H, Jin H, Chen Q, Pan J: The protective effect of Ellagic acid (EA) in osteoarthritis: An in vitro and in vivo study. BIOMED PHARMACOTHER 2020, 125:109845.

[46] Zupan J, Vrtačnik P, Cör A, Haring G, Weryha G, Visvikis-Siest S, Marc J: VEGF-A is associated with early degenerative changes in cartilage and subchondral bone. GROWTH FACTORS 2018, 36(5-6):263-273.

[47] Hamilton JL, Nagao M, Levine BR, Chen D, Olsen BR, Im HJ: Targeting VEGF and Its Receptors for the Treatment of Osteoarthritis and Associated Pain. J BONE MINER RES 2016, 31(5):911-924.

[48] Vadalà G, Russo F, Musumeci M, Giacalone A, Papalia R, Denaro V: Targeting VEGF-A in cartilage repair and regeneration: state of the art and perspectives. J Biol Regul Homeost Agents 2018, 32(6 Suppl. 1):217-224.

[49] Zan PF, Yao J, Wu Z, Yang Y, Hu S, Li GD: Cyclin D1 Gene Silencing Promotes IL-1ß-Induced Apoptosis in Rat Chondrocytes. J CELL BIOCHEM 2018, 119(1):290-299.

[50] Zou J, Li XL, Shi ZM, Xue JF: Effects of C-myc gene silencing on interleukin-1ß-induced rat chondrocyte cell proliferation, apoptosis and cytokine expression. J BONE MINER METAB 2018, 36(3):286-296.

[51] Rhee J, Park SH, Kim SK, Kim JH, Ha CW, Chun CH, Chun JS: Inhibition of BATF/JUN transcriptional activity protects against osteoarthritic cartilage destruction. ANN RHEUM DIS 2017, 76(2):427-434. 
[52] Teng P, Liu Y, Dai Y, Zhang H, Liu WT, Hu J: Nicotine Attenuates Osteoarthritis Pain and Matrix Metalloproteinase-9 Expression via the a7 Nicotinic Acetylcholine Receptor. J IMMUNOL 2019, 203(2):485-492.

[53] Jackson MT, Moradi B, Smith MM, Jackson CJ, Little CB: Activation of matrix metalloproteinases 2, 9, and 13 by activated protein C in human osteoarthritic cartilage chondrocytes. ARTHRITIS RHEUMATOL 2014, 66(6):1525-1536.

[54] Xue M, McKelvey K, Shen K, Minhas N, March L, Park SY, Jackson CJ: Endogenous MMP-9 and not MMP-2 promotes rheumatoid synovial fibroblast survival, inflammation and cartilage degradation. Rheumatology (Oxford) 2014, 53(12):2270-2279.

[55] Eskelinen A, Tanska P, Florea C, Orozco GA, Julkunen P, Grodzinsky AJ, Korhonen RK: Mechanobiological model for simulation of injured cartilage degradation via pro-inflammatory cytokines and mechanical stimulus. PLOS COMPUT BIOL 2020, 16(6):e1007998.

[56] Zevenbergen L, Gsell W, Chan DD, Vander SJ, Himmelreich U, Neu CP, Jonkers I: Functional assessment of strains around a full-thickness and critical sized articular cartilage defect under compressive loading using MRI. Osteoarthritis Cartilage 2018, 26(12):1710-1721.

[57] Myller K, Korhonen RK, Töyräs J, Salo J, Jurvelin JS, Venäläinen MS: Computational evaluation of altered biomechanics related to articular cartilage lesions observed in vivo. J ORTHOP RES 2019, 37(5):1042-1051.

[58] Wang T, He C: Pro-inflammatory cytokines: The link between obesity and osteoarthritis. Cytokine Growth Factor Rev 2018, 44:38-50.

[59] Liao CR, Wang SN, Zhu SY, Wang YQ, Li ZZ, Liu ZY, Jiang WS, Chen JT, Wu Q: Advanced oxidation protein products increase TNF-a and IL-1 $\beta$ expression in chondrocytes via NADPH oxidase 4 and accelerate cartilage degeneration in osteoarthritis progression. REDOX BIOL 2020, $28: 101306$.

[60] Sun K, Luo J, Guo J, Yao X, Jing X, Guo F: The PI3K/AKT/mTOR signaling pathway in osteoarthritis: a narrative review. Osteoarthritis Cartilage 2020, 28(4):400-409.

[61] Ke H, Mou X, Xia Q: Remifentanil repairs cartilage damage and reduces the degradation of cartilage matrix in post-traumatic osteoarthritis, and inhibits IL-1ß-induced apoptosis of articular chondrocytes via inhibition of PI3K/AKT/NF-KB phosphorylation. Ann TransI Med 2020, 8(22):1487.

\section{Figures}




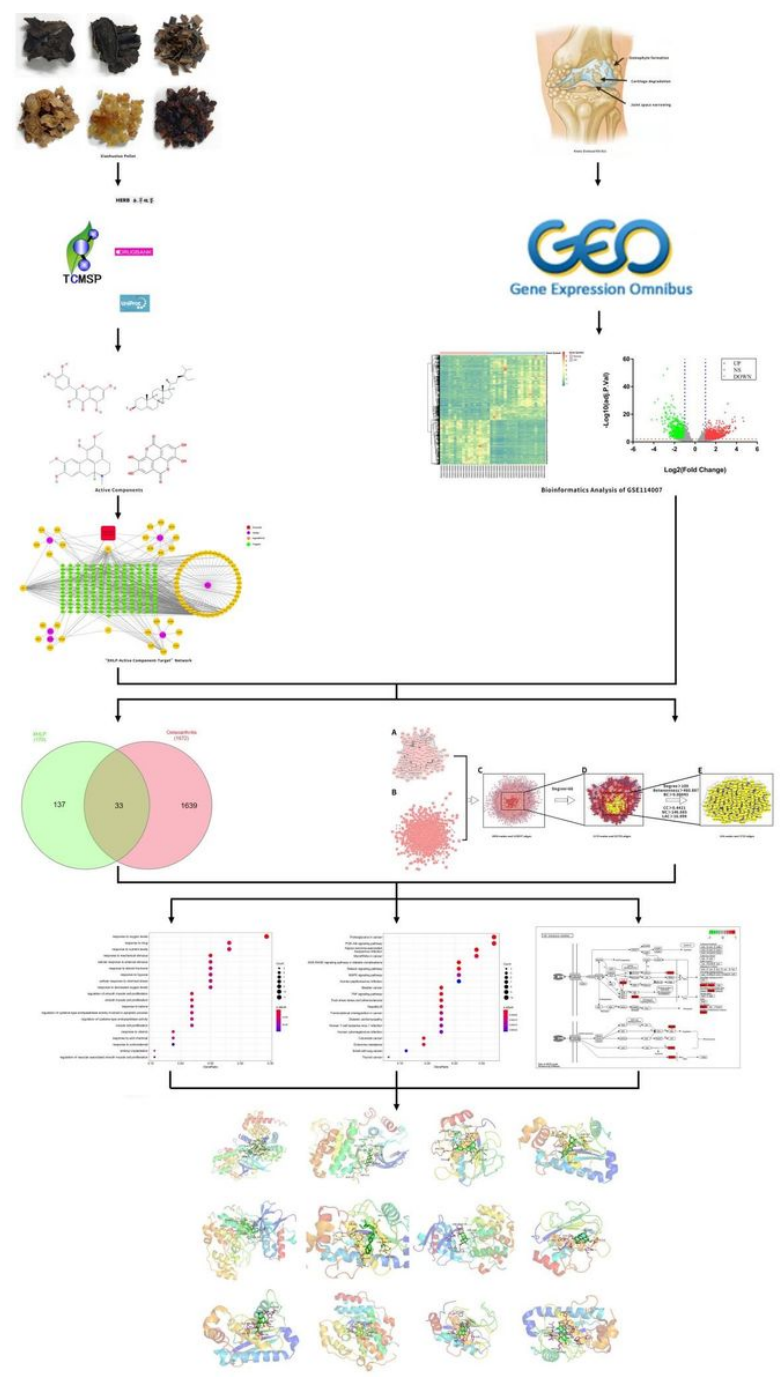

Figure 1

Schematic diagram for identifying the mechanism of XHLP anti-Osteoarthritis by bioinformatics analysis. 


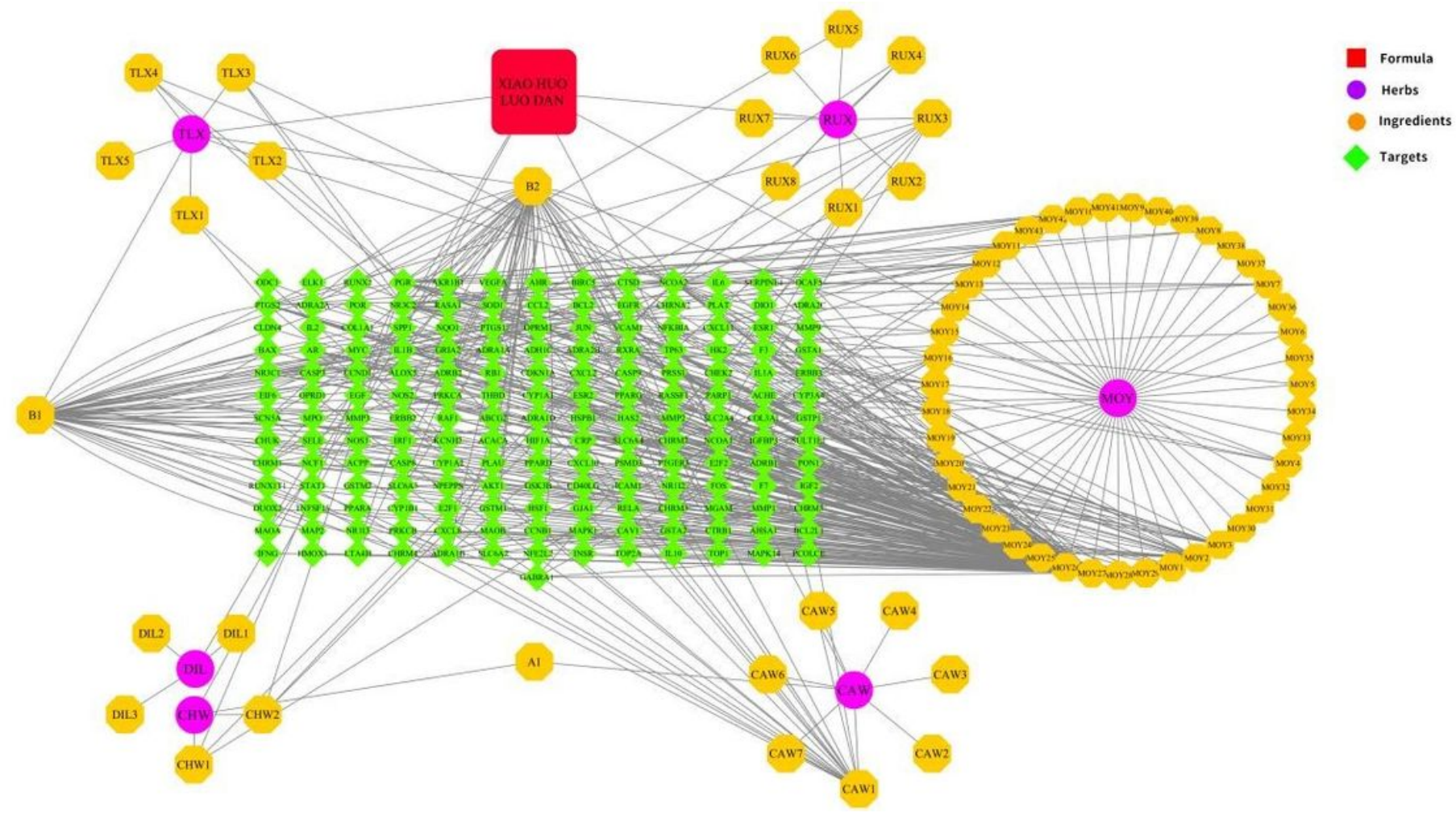

Figure 2

"XHLP-Active Component-Target" Network: the purple circles in the network represent the drugs in XHLP, the orange hexagons represent the active components, and the green diamonds represent the target genes. 
A

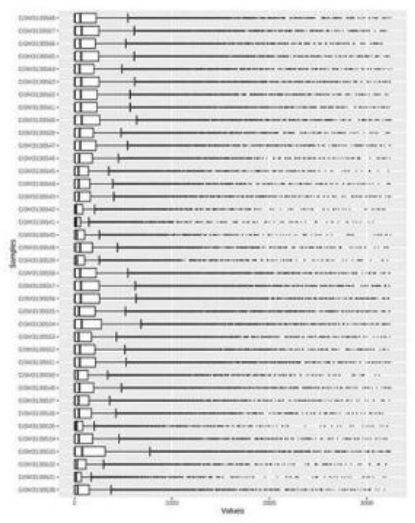

Filtering: Variance filter $=15.0$, Low abundance $=4.0$

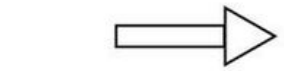

Normalization: Log2-counts per million

Filtering: Variance filter $=15.0$, Low abundance $=4.0$

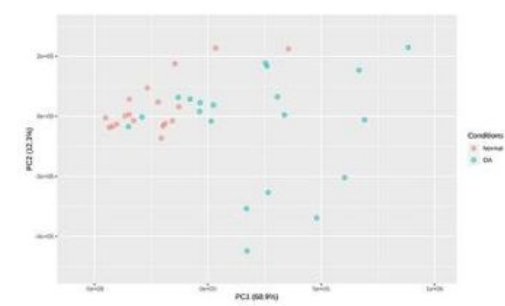

C

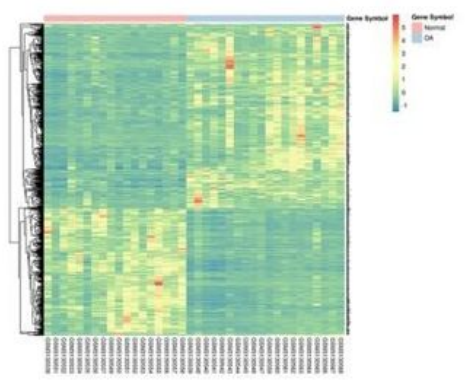

D

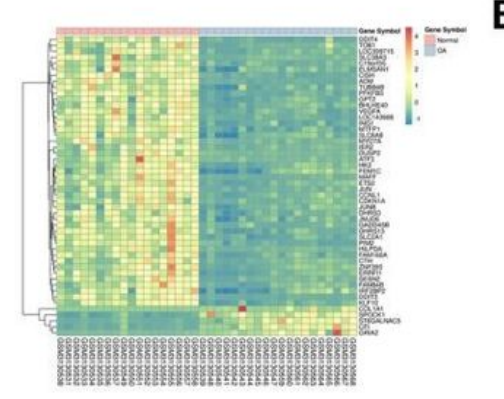

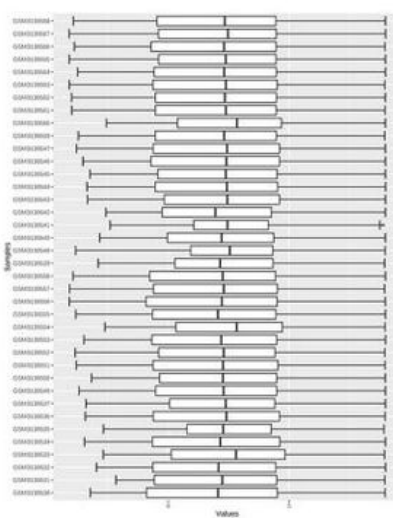

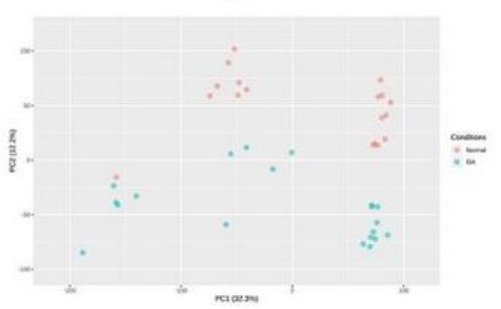

E

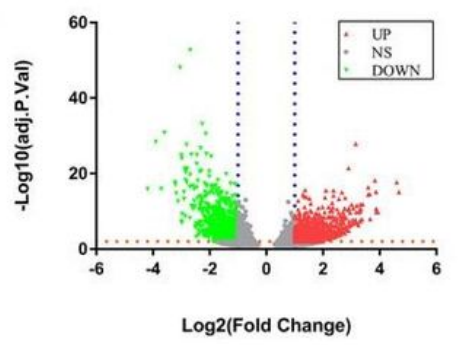

Figure 3

Bioinformatics Analysis of GSE114007. (A) Box plots of samples before and after normalization, representing the counts distribution of each sample; (B)PCA plot before and after normalization; each point represents a sample; the farther the distance between the two samples, the greater the difference

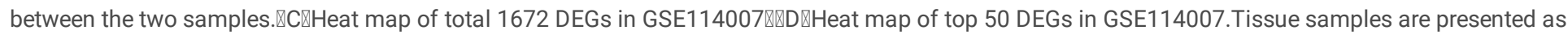
columns; individual genes are presented as rows. Red indicates upregulated genes and green indicates downregulated genes in patients with KOA. The patients in the top rows in pink indicates columns were normals; the patients in the top rows in blue indicates columns were patients with KOA. $\mathbb{E} \triangle \mathrm{The}$ volcano plot of total 1672 DEGs in GSE114007. The vertical Blue lines correspond to Log2FC up and down and the horizontal Orange line represents an adjusted P-value of 0.01 . The Red point represents the differentially upregulated genes with statistical significance.The Green point represents the differentially downregulated genes with statistical significance. 
A

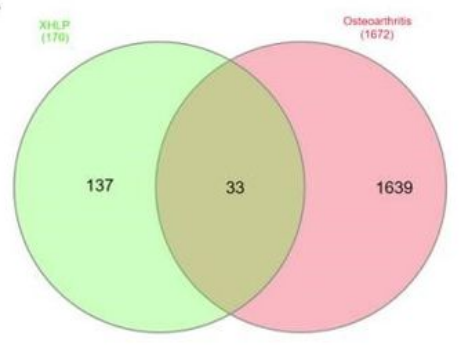

B

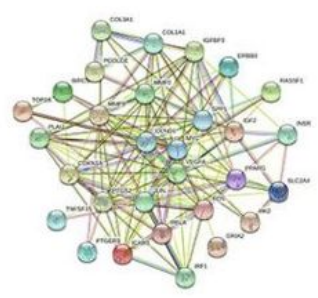

C

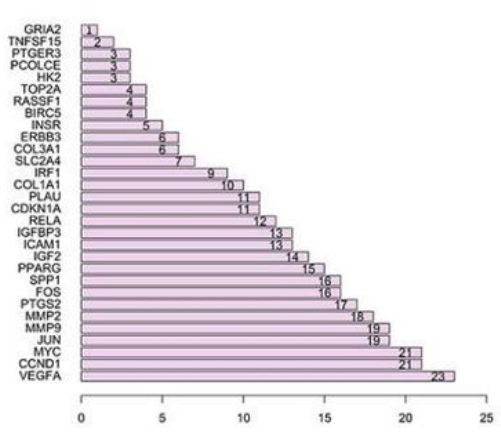

D

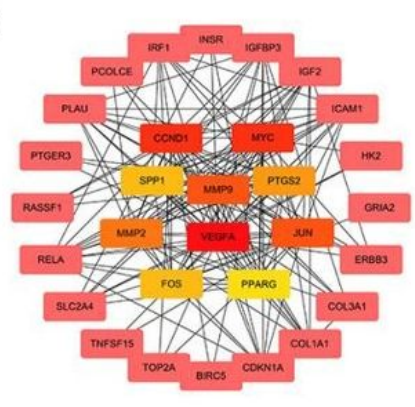

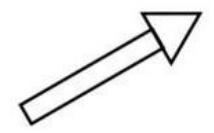

E
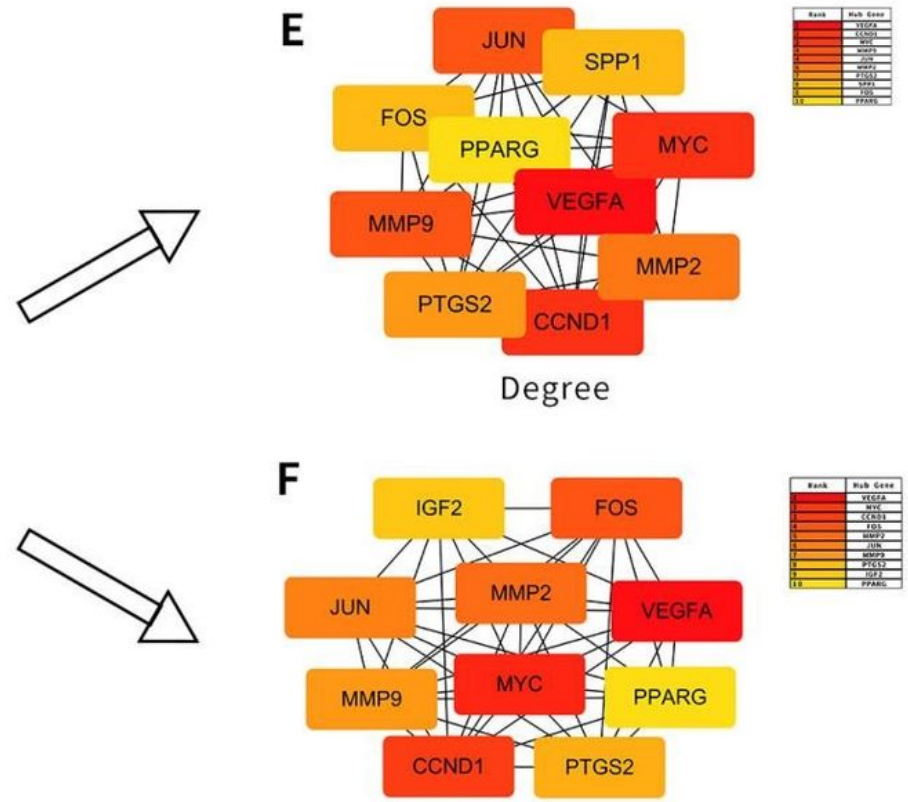

Betweenness

\section{Figure 4}

"XHLP-KOA-Common Targets"Network Analysis.(A)Venn diagram of KOA-related targets of XHLP into the active components.(B)PPI network generated on XHLP-KOA mapped targets.(C) Top 30 candidate targets extracted from PPI according to the node degree.(D)PPI network diagram of KOA joint targets treated with XHLP.(E)PPI network of the top 10 hub proteins of co-targets based on Degree centrality.(F)PPI network of the top 10 hub proteins of cotargets based on Betweenness centrality.

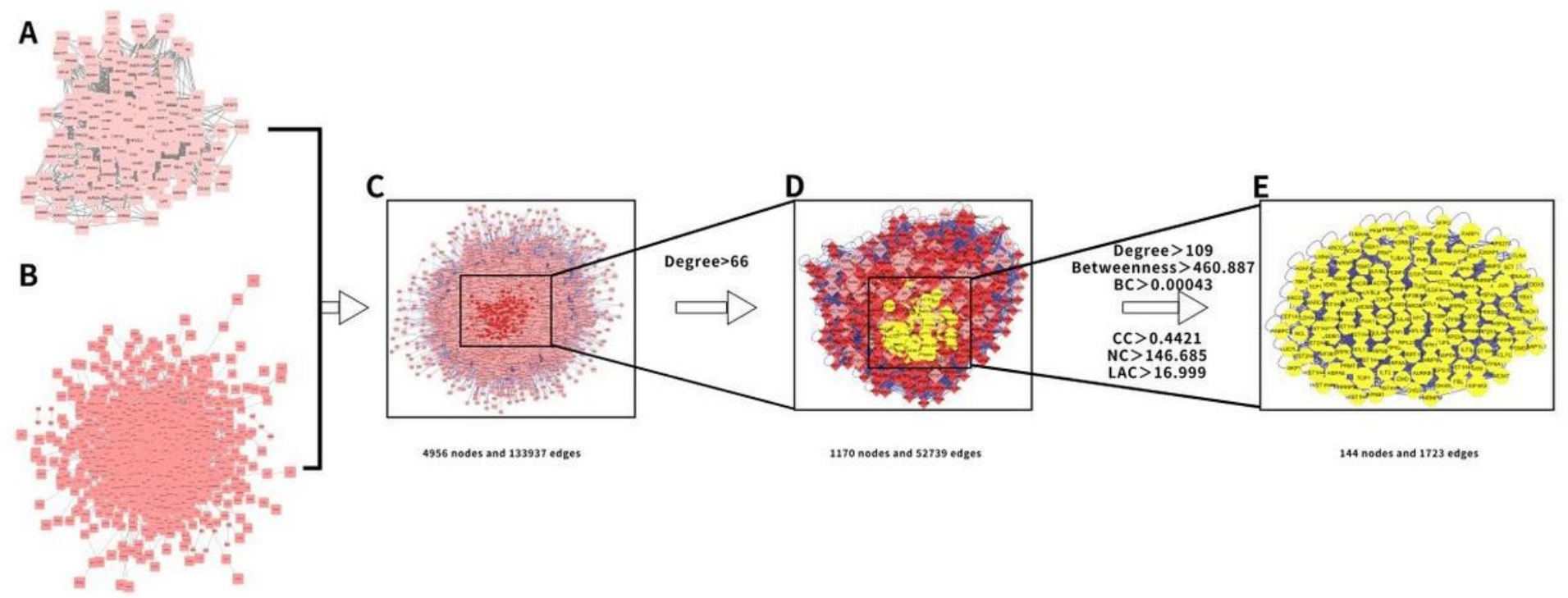

Figure 5 
Process of topological screening for the PPI network.(A)PPI network of XHLP targets.(B)PPI network of KOA differentially expressed genes.(C)PPI network of key targets of merging mapping.(D)PPI network of key genes with a screening threshold of Degree>66 (2 times the median value of Degree).(E)PPI

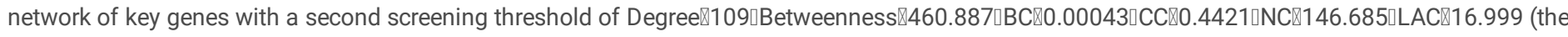
median value of all the above).

A

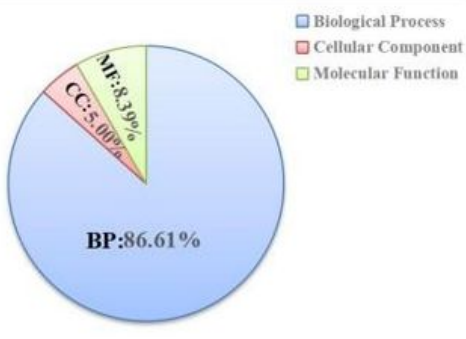

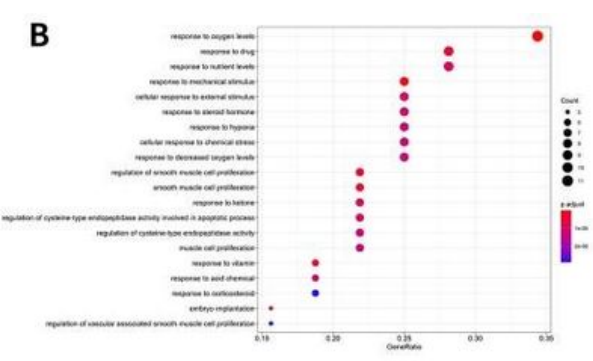
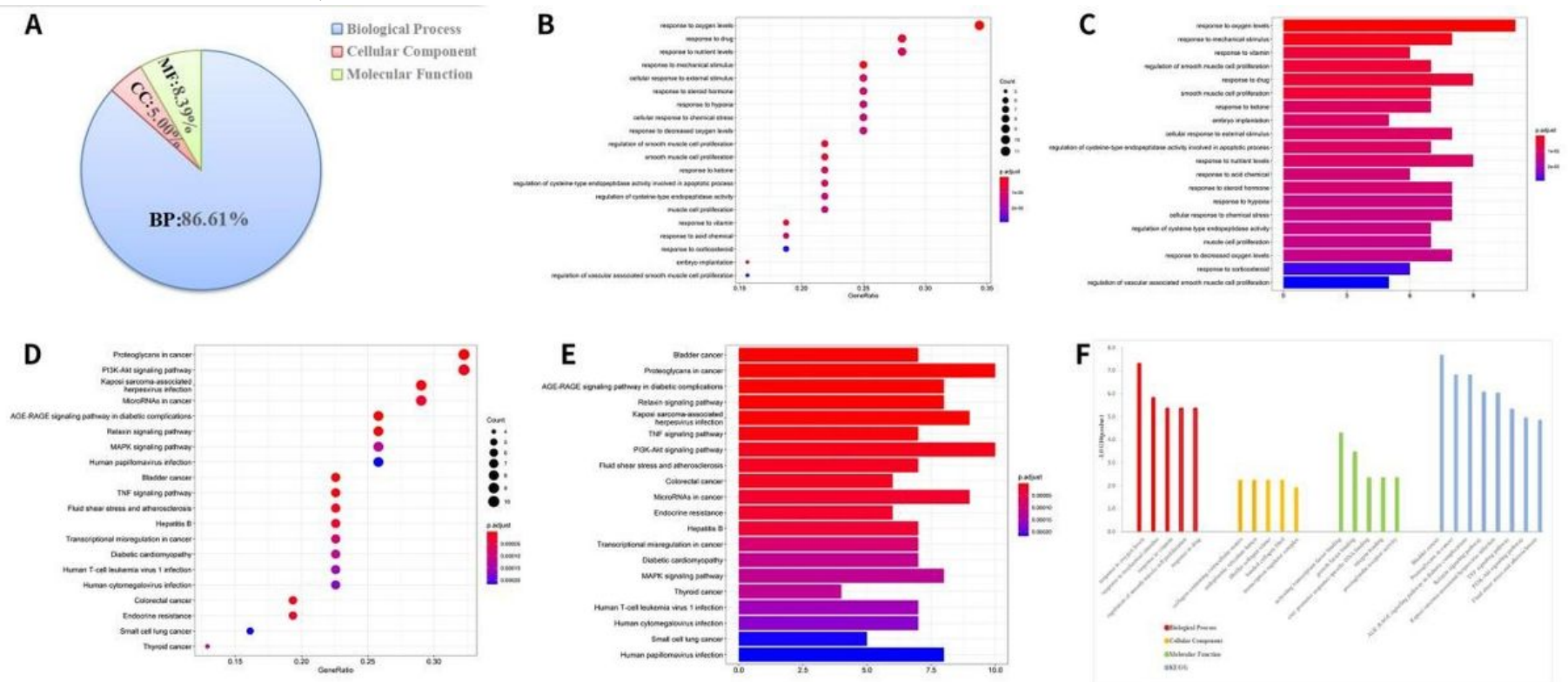
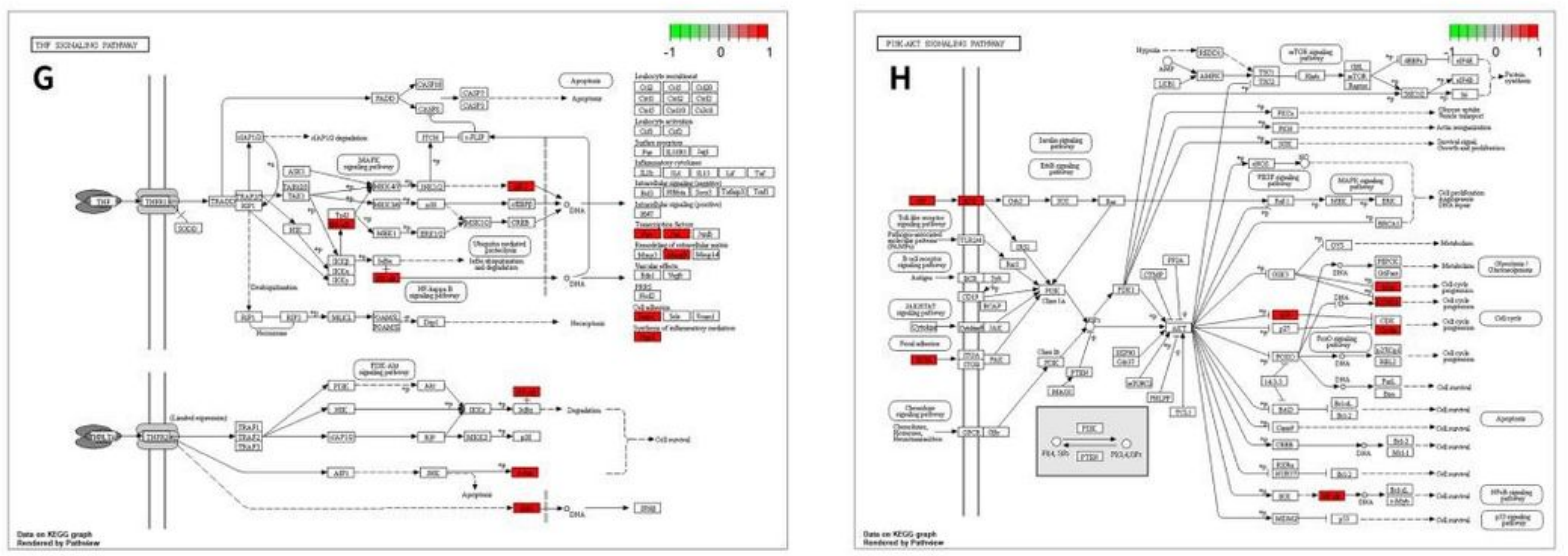

Figure 6

GO and KEGG enrichment analyses of 33 candidate targets.(A)Three types of GO terms for target genes and gene products.(B)the Bubble chart of the top 20 terms extracted accroding to the p.adjust value based on GO enrichment analysis.(C)the Bar chart of the top 20 terms extracted accroding to the p.adjust value based on GO enrichment analysis.(D)the Bubble chart of the top 20 pathways extracted accroding to the p.adjust value based on KEGG enrichment analysis.(E)the Bar graph of the top 20 pathways extracted accroding to the p.adjust value based on KEGG enrichment analysis.(F)Top 5 significantly enriched BP,CC, and MF categories based on gene ontology,and the Top 8 significantly enriched BP,CC, and MF categories.(G)TNF signaling pathway map of KOA targets treated with XHLP.(H)PI3K-Akt signaling pathway map of KOA targets treated with XHLP.(the red nodes represent the most potential targets,arrows represent the activation effect, T arrows represent the inhibition effect, and segments show the activation effect or inhibition effect.) 

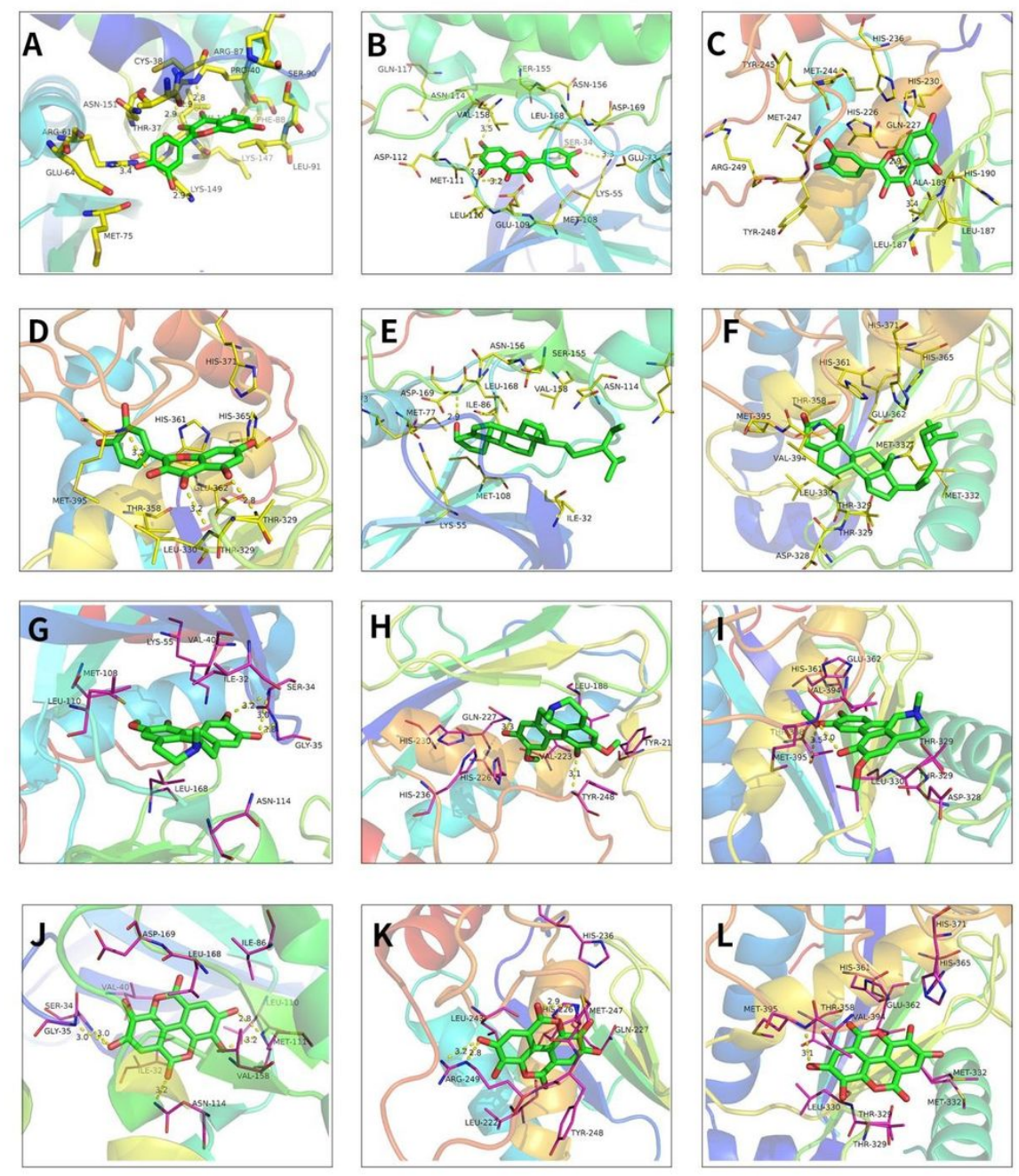

\section{Figure 7}

3D Molecular docking model of the 5 key active ingredients with the 6 hub-targets.(A)The molecular docking of quercetin with CCND1(2W96);(B)The molecular docking of quercetin with JUN(3V3V);(C)The molecular docking of quercetin with MMP9(6ESM); (D)The molecular docking of quercetin with MMP2(4WK7); (E)The molecular docking of beta-sitosterol with JUN(3V3V);(F)The molecular docking of beta-sitosterol with MMP2(4WK7);(G)The molecular docking of Izoteolin with JUN(3V3V);(H)The molecular docking of Izoteolin with MMP9(6ESM); (I)The molecular docking of Izoteolin with MMP2(4WK7); (J)The molecular docking of ellagic acid with JUN(3V3V);(K)The molecular docking of ellagic acid with MMP9(6ESM);(L)The molecular docking of ellagic acid with MMP2(4WK7). 

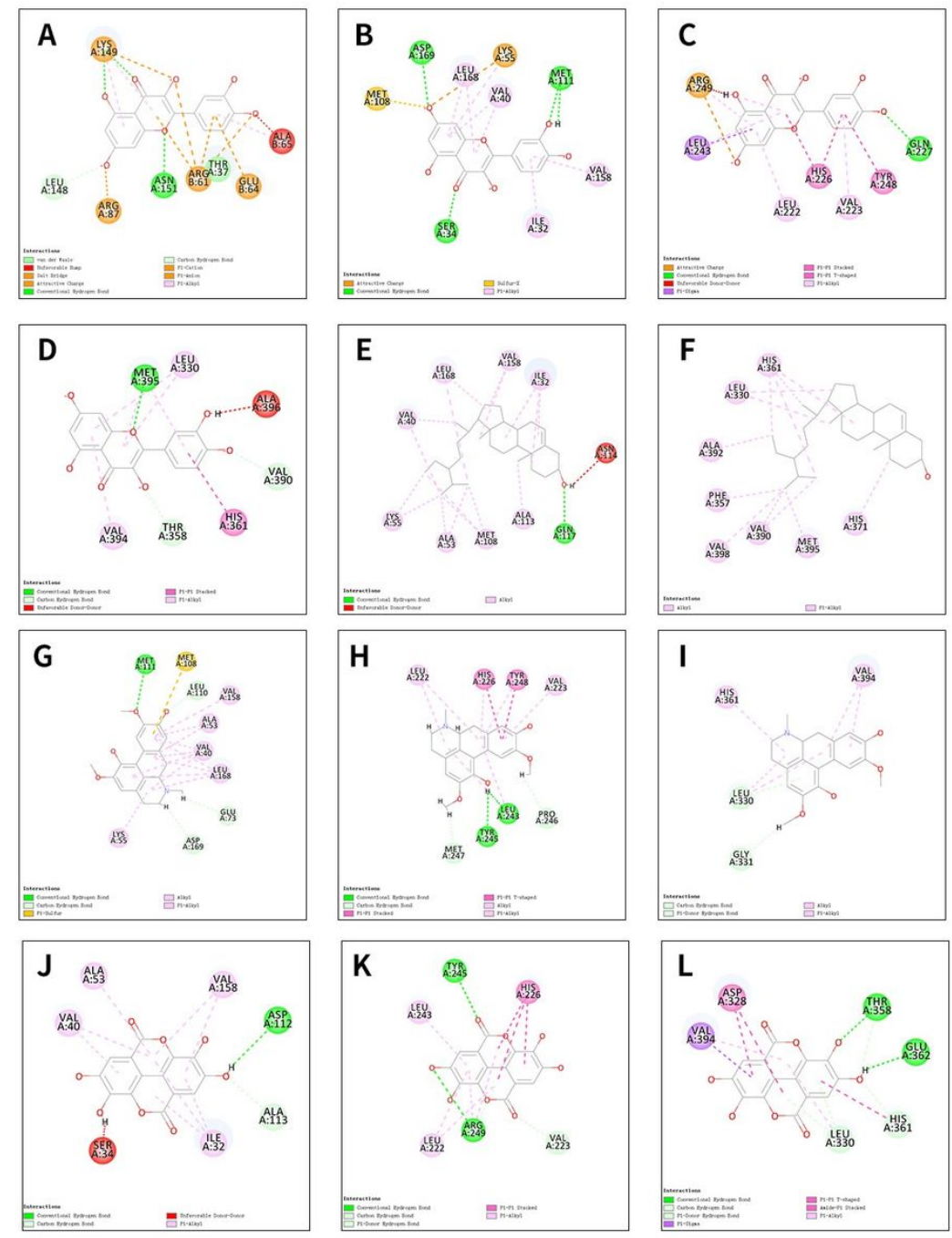

\section{Figure 8}

2D Virtual Molecular docking of bioactive ingredients from XHLP for Knee Osteoarthritis targets.(A)The molecular docking of quercetin with CCND1(2W96);(B)The molecular docking of quercetin with JUN(3V3V);(C)The molecular docking of quercetin with MMP9(6ESM); (D)The molecular docking of quercetin with MMP2(4WK7); (E)The molecular docking of beta-sitosterol with JUN(3V3V);(F)The molecular docking of beta-sitosterol with MMP2(4WK7);(G)The molecular docking of Izoteolin with JUN(3V3V);(H)The molecular docking of Izoteolin with MMP9(6ESM); (I)The molecular docking of Izoteolin with MMP2(4WK7); (J)The molecular docking of ellagic acid with JUN(3V3V);(K)The molecular docking of ellagic acid with MMP9(6ESM); (L)The molecular docking of ellagic acid with MMP2(4WK7). 